\title{
ASYMPTOTIC VALUES OF MEROMORPHIC FUNCTIONS OF FINITE ORDER
}

\author{
A. CANTÓN, D. DRASIN AND A. GRANADOS
}

\begin{abstract}
The asymptotic values of a meromorphic function (of any order) defined in the complex plane form a Suslin-analytic set. Moreover, given an analytic set $A^{*}$ we construct a meromorphic function of finite order and minimal growth having $A^{*}$ as its precise set of asymptotic values.
\end{abstract}

\section{INTRODUCTION}

A nonconstant meromorphic function $f(z)$ in the plane has the asymptotic value $a$ if there is a curve $\gamma$ tending to $\infty$ such that $f(z) \rightarrow a$ as $z \rightarrow \infty, z \in \gamma$. Let $A s(f)$ be the set of asymptotic values of $f$; for example, $A s\left(e^{z}\right)=\{0, \infty\}$. A classical result of Mazurkiewicz [13] asserts that $A s(f)$ is an analytic set in the sense of Suslin $[3,16]$.

Recall that the order of $f$ is given by

$$
\lambda=\limsup _{r \rightarrow \infty} \frac{\log T(r, f)}{\log r},
$$

where $T(r, f)$ is the Nevanlinna characteristic (when $f$ is entire, $T(r, f)$ may be replaced by $\log M(r, f)$, with $M(r, f)$ the maximum modulus function).

Heins [11] showed that given an analytic set $A^{*}$, there is a meromorphic function $f$ with $A s(f)=A^{*}$ and, if $\infty \in A^{*}$, then $A^{*}=A s(f)$ for some entire function $f$. In general, Heins's function has infinite order. For example, if

$$
A:=A^{*} \backslash\{\infty\}=A^{*} \cap \mathbb{C},
$$

and card $(A)=\infty$ with $A$ bounded, Heins produces a Riemann surface with infinitely many 'logarithmic branch points' over $w=\infty$, so by Ahlfors's theorem $\lambda=\infty$. Note that $A$, as the intersection of two analytic sets, is analytic.

Eremenko [8] produced meromorphic functions with $\lambda<\infty$ having $A s(f)=\hat{\mathbb{C}}$. In fact, if $\psi(r)$ is a given increasing unbounded function, he could arrange that

$$
T(r, f)<\psi(r) \log ^{2} r \quad \text { as } r \rightarrow \infty,
$$

Date: March 4, 2010.

2000 Mathematics Subject Classification. Primary 30D30; Secondary 30D40, 30D35.

The first author benefited from a postdoctoral fellowship from the Ministerio de Educación, Cultura y Deportes (Spain) while visiting the University of Washington and a RED grant from La Generalitat de Catalunya while staying at the Universitat Autònoma de Barcelona. The third author benefited from a postdoctoral fellowship from the Ministerio de Educación, Cultura y Deportes (Spain) while visiting the University of Washington under the sponsorship of a Fulbright grant. The first and third authors were partially suppported by MTM2006-11976. Much of this research was performed at Purdue University, and the authors thank the Purdue Mathematics Department for its hospitality. 
and so $f$ even has order 0 . The significance of condition (2) is that when $\psi(r)=$ $O(1)$, Valiron [17] showed that $A s(f)$ contains at most one element.

Theorem 1. Given an analytic set $A^{*}$ in $\hat{\mathbb{C}}$ and $\lambda, 0 \leq \lambda \leq \infty$, there is a function $f$ meromorphic in the plane of order $\lambda$ such that

$$
A s(f)=A^{*} .
$$

Indeed, given an increasing function $\psi: \mathbb{R}^{+} \rightarrow \mathbb{R}^{+}, \psi(r) \rightarrow \infty$ as $r \rightarrow \infty$, one can arrange that $f$ satisfy (2).

Although questions of this type have been considered in various contexts for many years, the definitive result for meromorphic functions requires additional tools. Our final function $f$ appears only indirectly, although the structure of the asymptotic curves and the asymptotic values assigned to them is presented explicitly. In contrast to [8], a full chapter $(\S 4)$ is needed to show that no other asymptotic values occur, and it requires new techniques. In [6] there is an informal outline of this work, and full details are given here.

Since there are elementary examples with $A^{*}$ being empty or having one element, we assume $A^{*}$ has cardinality at least two, and $0, \infty \in A^{*}$. A key step is to produce a meromorphic function $g(z)$ whose growth also satisfies (2), with $A s(g)=\{0, \infty\}$, with data on the curves on which $g$ tends to its asymptotic values. We then follow ideas going back to Teichmüller and apply quasiconformal compositions to convert $g$ (via the Beltrami equation) to a meromorphic $f$ having $A s(f)=A^{*}$ with growth (2); even here, in $\S 5$ we must reformulate the standard definition of analytic set.

This meromorphic function $g$ arises by approximating a specific $\delta$-subharmonic function $U(z)$. The general form of $U$ is very simple, based on the fact that the function $u(z):=A+B \theta$ (with $\theta=\arg z$ ) is harmonic, and, if $B \neq 0$, of least growth. In $\S 2$ we introduce a simple model (called $\hat{U}$ here) whose inadequacies then point to the correct form of $U$ in $\S 2.2$. Although our final function is necessarily complicated, the analysis in $\S 4.3$ is based on studying the elementary function $w=\sin z$ (despite that $A s(\sin z)=\varnothing)$.

Throughout, $C$ is a finite positive constant which may change from line to line, unless specified otherwise, although the constants $C^{\prime}$ and $C_{0}$ introduced in (18) and Theorems 2 and $2^{\prime}$ are absolute, associated to the data $\left\{A^{*}, \psi\right\}$ of (1) and (2). In addition to $\mathcal{U}=\{z: \Im z>0\}, S(r)=\{|z|=r\}$, we set $B(a, r)=\{|z-a|<r\}$, $B(r)=B(0, r), \stackrel{\circ}{E}$ the interior of $E, \bar{E}$ its closure, and $a \wedge b=\min (a, b)$.

Acknowledgments. We would like to thank the referee for helpful comments and suggestions.

\section{The FUNCTION $U$ AND ITS LAPLACIAN}

2.1. The toy function $\hat{U}$. We introduce $\hat{U}(z)$, a simplified version of $U$, first for $z$ in the upper half-plane $\mathcal{U}$. Take $0=\Theta_{0}<\Theta_{1}<\cdots<\Theta_{k}<\Theta_{k+1}=\pi$ with data $L>0$, boundary values $\hat{U}(r)=\hat{U}\left(r e^{i \pi}\right)=0(r>0)$ and constant values $\hat{U}\left(r e^{i \Theta_{\ell}}\right)$ on the system of rays $\arg z=\Theta_{\ell}, 1 \leq \ell \leq k$, in $\mathcal{U}$. Then for $r>0,0 \leq \theta \leq \pi$, extend $\hat{U}$ to each sector $\left\{\Theta_{\ell}<\arg z<\Theta_{\ell+1}\right\}, 0 \leq \ell \leq k$, by

$$
\hat{U}\left(r e^{i \theta}\right)=\min \left\{\hat{U}\left(r e^{i \Theta_{\ell}}\right)+L\left(\theta-\Theta_{\ell}\right), \hat{U}\left(r e^{i \Theta_{\ell+1}}\right)+L\left(\Theta_{\ell+1}-\theta\right)\right\} .
$$


In what follows it will be assumed that data $\hat{U}\left(r e^{i \theta}\right)$ are chosen so that (3) defines $\Psi_{\ell+1} \in\left[\Theta_{\ell}, \Theta_{\ell+1}\right],(0 \leq \ell \leq k)$ as the $\theta$-value at which each pair of linear functions coincide, and $\hat{U}$ has a local maximum in $\theta$ at each $\Psi_{\ell+1}$. Thus $\hat{U}$ is piecewise-linear function in $\theta$, vanishes on the real axis (other that at $z=0$ where it is not defined), and monotonic on each $\theta$-interval $\left\{\Theta_{\ell}<\theta<\Psi_{\ell+1}\right\},\left\{\Psi_{\ell+1}<\theta<\Theta_{\ell+1}\right\}, 0 \leq \ell \leq k$. Figure 1 shows one possible graph on $[0, \pi]$ with $k=3$.

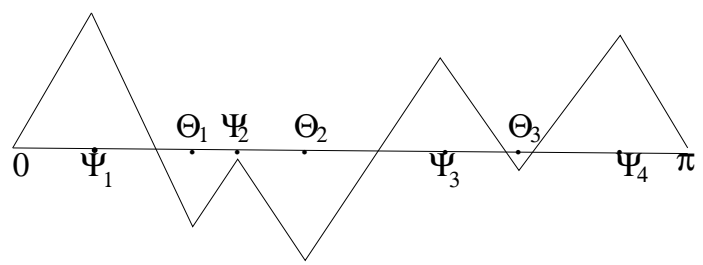

FiguRE 1. Graph of $\hat{U}\left(r e^{i \theta}\right)$ for fixed $r$.

The function $\hat{U}$ of (3) is $\delta$-subharmonic in $\mathcal{U}$ (i. e., $\Delta \hat{U}$ is a signed measure (charge)), zero on $\partial \mathcal{U} \backslash\{0\}$, and harmonic off the rays $\left\{\arg z=\Theta_{\ell}, \Psi_{\ell}\right\}$, and so may be extended to be $\delta$-subharmonic on $\mathbb{C} \backslash\{0\}$ by

$$
\hat{U}(-z)=-\hat{U}(z) \quad(z \in \mathcal{U})
$$

a rigidity we use henceforth, and without which the approximation arguments (§4) would collapse $((4)$ is the key to $(37))$. It also produces respectively $k+1$ and $k$ rays in the lower half plane on which $\hat{U}$ has local minima and maxima (in $\theta$ ) on $S(r)$. For any function $\hat{U}$ considered here (or, later, $U$ ), let $\Gamma^{0}$ be the curves which are the locus of local minima in $\theta$ of $\hat{U}\left(r e^{i \theta}\right)$ for fixed $r>0, \Gamma^{*}$ those which are the locus of local maxima, and

$$
\Gamma^{\sharp}:=\Gamma^{0} \cup \Gamma^{*} \text {. }
$$

Thus for $\hat{U}, \Gamma^{\sharp}$ is a network of $4 k+2$ rays, with $\Gamma^{\sharp} \cap(\mathbb{R} \backslash\{0\})=\varnothing$.

The Laplacian of $\hat{U}$ has a special nature (at least if $z \neq 0$ ): $\Delta \hat{U}(z)=0$ when $z=r e^{i \theta} \notin \Gamma^{\sharp}$, whereas if $z \in \Gamma^{\sharp} \cup S(r)$, the formula $\Delta u=u_{r r}+r^{-1} u_{r}+r^{-2} u_{\theta \theta}$ shows that if $z=r e^{i \theta}$, then

$$
\Delta \hat{U}\left(r e^{i \theta}\right)= \pm 2 L r^{-2} \delta_{\varphi}(\theta),
$$

where $\delta_{\varphi}(\theta)$ is the Dirac function; the plus sign is used when $z \in \Gamma^{0}$, and the minus sign when $z \in \Gamma^{*}$ (much as $\left.|x|^{\prime \prime}=2 \delta_{0}\right)$. In summary,

$$
\Delta \hat{U}\left(r e^{i \theta}\right)=2 r^{-2} L\left[\sum_{\theta^{0} \in \Gamma^{0}} \delta_{\theta^{0}}(\theta)-\sum_{\theta^{*} \in \Gamma^{*}} \delta_{\theta^{*}}(\theta)\right] .
$$

To obtain a meromorphic function $\hat{g}$ such that $\log |\hat{g}(z)| \operatorname{mimics} \hat{U}(z)$, we approximate $\Delta \hat{U}$ by a measure composed of (positive and negative) unit masses, the principle being that $(a)$ if $\Delta v$ is a Borel measure consisting exclusively of unit point masses, then $v=\log |\hat{g}|$ for some meromorphic function $\hat{g}$, and $(b)$ we can recover the asymptotic behavior of $\hat{g}$ from graphs as in Figure 1 at points at which $|\hat{U}(z)-\log | \hat{g}(z)||$ is small. We see later (Lemma 9) that $g$ attains its asymptotic values on curves in $\Gamma^{\sharp}$, but probably not on all curves. 
2.2. What is wrong with $\hat{U}$ ? Suppose $\hat{g}$ is meromorphic with $\log |\hat{g}|$ modelled on $\hat{U}$ using (3) in $\mathcal{U}$ and (4) in $\mathbb{C} \backslash \mathcal{U}$. For $r>0$, each $S(r) \cap \Gamma^{\sharp}$ has $2(2 k+1)$ points, so a straight forward computation in $\S 7.1$ (based on (5)) will show that

$$
T(r, \hat{g})=(4 k+2+o(1)) \log ^{2} r(r \rightarrow \infty) .
$$

Thus $T(r, \hat{g}) / \log ^{2} r$ is bounded and in fact since $\hat{U}$ is bounded, we could not expect 0 or $\infty$ to be asymptotic values of $g$. To circumvent this, our function $U$ is a 'limit' of functions $\hat{U}$ as $k, L \uparrow \infty$. Then on each $S(r) \cap \mathcal{U}$, the graph of $U\left(r e^{i \theta}\right)$ will be as in Figure 1, but with complexity increasing with $r$, in a manner that

$$
\lim _{r \rightarrow \infty} \inf _{S(r)} U(z)=-\infty, \quad \lim _{r \rightarrow \infty} \sup _{S(r)} U(z)=+\infty .
$$

The meromorphic function $g$ for which $\log |g|$ approximates $U$ is obtained by 'atomizing' $\Delta U$ exactly as described in $\S 2.1$ for $\hat{U}$.

We partition $\mathbb{C}$ into the disk $\mathcal{A}_{0}=\left\{|z|<r_{0}\right\}$ and annuli $\mathcal{A}_{k}$,

$$
\mathcal{A}_{k}:=\left\{r_{k-1} \leq|z|<r_{k}\right\} \quad(k \geq 1),
$$

for a rapidly-increasing sequence $\left\{r_{k}\right\}$ with $r_{0}>1$. The function $U$ is defined on $\mathbb{C}$ so that relative to each $\mathcal{A}_{k}$ it mimics a toy function $\hat{U}$ of increasing complexity.

Thus, in place of the constant $L$ in (3), let $L(r) \uparrow \infty$ be a smooth function with $L(r)=0$ on $[0,1]$, and for is some fixed constant $C^{\prime}$, say $C^{\prime}=20$, suppose that

$$
\begin{aligned}
& \lim _{r \rightarrow \infty} r^{-1} L(r)+r L^{\prime}(r)+r^{2}\left|L^{\prime \prime}(r)\right|=0 \\
& \sup _{r>0} r^{-1} L(r)+r L^{\prime}(r)+r^{2}\left|L^{\prime \prime}(r)\right| \leq C^{\prime} .
\end{aligned}
$$

To satisfy (2), suppose that

$$
L^{2}\left(r^{21}\right)=o(\psi(r)) \quad(r \rightarrow \infty)
$$

(any large number would work in place of 21 ), and impose the compatability conditions

$$
\log \left(r_{k+1} / r_{k}\right)>\left(k+L_{0}\right) L\left(r_{k+1}\right) \text { and } L\left(r_{k}\right)>(k+1)^{3} \quad(k \geq 0),
$$

for some value $L_{0}$ large enough (for example taking $L_{0}>\left((5 / 4)^{2 / 3}-1\right)^{-1}$ gives constant 10 in (31))

Comment. Conditions such as (7) and, later, (15) play an important role. A helpful way to visualize them is to choose, for each $k$, suitable numbers $L_{k}$ and $\delta_{k}>0$. Then we may arrange that $L\left(r_{k}\right)-L\left(r_{k-1}\right)=L_{k}$ with $\sup _{\left[r_{k-1}, r_{k}\right]} r L^{\prime}(r)<\delta_{k}$ by increasing the ratio $r_{k} / r_{k-1}$ as needed. In turn, these conditions are compatible with $\sup _{\left[r_{k-1}, r_{k}\right]} r^{2}\left|L^{\prime \prime}(r)\right|$ being small, increasing $r_{k} / r_{k-1}$ if necessary.

Other restrictions will be given later. They will be of two types. Often the ratios $\left\{r_{k} / r_{k-1}\right\}$ will increase, but not the values $\left\{L\left(r_{k}\right)\right\}$, so that $(7)-(9)$ remain valid. In addition, $\S 2.5$ introduces additional conditions, many of which might be avoided at the expense of complicating several arguments.

2.3. Graph of $\mathbf{U}$. Our fundamental function $U$ is modelled on (3) in each $\mathcal{A}_{k}$. For $z \in \mathcal{U} \cap \mathcal{A}_{0}$, first set

$$
U\left(r e^{i \theta}\right)=L(r) \min \{\theta, \pi-\theta\} \quad\left(0 \leq r \leq r_{0}, 0 \leq \theta \leq \pi\right),
$$

and then use (4) on $\mathcal{A}_{0} \backslash \mathcal{U}$. Since $L(r) \equiv 0$ on $[0,1], U(z) \equiv 0$ for $z \in B(1)$. Define,

$$
U\left(r_{0} e^{i \theta}\right)=L\left(r_{0}\right)(\min \{\theta, \pi-\theta\}) \quad(0 \leq \theta \leq \pi)
$$


on $B\left(r_{0}\right) \cap \mathcal{U}=\partial \mathcal{A}_{0} \cap \mathcal{U}$. When $\theta=\pi / 2, \theta=\pi-\theta$ so that $\Gamma^{\sharp} \cap\left(\mathcal{U} \cap B\left(r_{0}\right)\right)=$ $\Gamma^{*} \cap\left(\mathcal{U} \cap B\left(r_{0}\right)\right)=\left\{r e^{i \pi / 2}, 1 \leq r \leq r_{0}\right\}$, and $z_{0}=i r_{0}$ will be the initial point of $\Gamma^{0} \cap \mathcal{U}$.

Since $U=0$ on $\mathbb{R}$, and $U$ is odd (see (4)) we need only define $U$ on $\mathcal{U} \cap\left\{|z| \geq r_{0}\right\}$. In fact, relative to $\mathcal{A}_{k}, k \geq 1, U$ will depend on how it is specified on the arcs of $\Gamma^{0} \cap\left(\mathcal{A}_{k} \cap \mathcal{U}\right)$. Hence (see Figure 1 or Figure 2), for each $k \geq 1$, mark $k$ arguments $\Theta_{\ell}$ on each of the two arcs of $\partial \mathcal{A}_{k} \cap \mathcal{U}$ augmented by $\Theta_{0}=0, \Theta_{k+1}=\pi$, with

$$
\begin{aligned}
& 0=\Theta_{0}^{-}(k)<\Theta_{1}^{-}(k) \leq \cdots \leq \Theta_{k}^{-}(k)<\Theta_{k+1}^{-}(k)=\pi \in S\left(r_{k-1}\right), \\
& 0=\Theta_{0}^{+}(k)<\Theta_{1}^{+}(k)<\cdots<\Theta_{k}^{+}(k)<\Theta_{k+1}^{+}(k)=\pi \in S\left(r_{k}\right) .
\end{aligned}
$$

Relative to $\mathcal{A}_{k} \cap \mathcal{U}$, the $k$ arcs of $\Gamma^{0}$ joining its boundary components connect $r_{k-1} e^{i \Theta_{\ell}^{-}(k)}$ to $r_{k} e^{i \Theta_{\ell}^{+}(k)}, 1 \leq \ell \leq k$. Since $S\left(r_{k}\right)=\partial \mathcal{A}_{k} \cap \partial \mathcal{A}_{k+1}$, we require for $k \geq 2$ that the sets

$$
\left\{\Theta_{\ell}^{-}(k)\right\}=\left\{\Theta_{\ell}^{+}(k-1)\right\},
$$

which with the second line of $(11)$ forces $\Theta_{\ell}^{-}(k)=\Theta_{\ell+1}^{-}(k)$ for (at least) one $1 \leq$ $\ell=\ell(k) \leq k$; see (26) and Figure 2. (Notice the strict inequalities of the first line of $(11))$.

Now suppose some given values are assigned to each of the points

$$
U\left(r_{p} e^{i \Theta_{\ell}^{+}(k)}\right), \quad U\left(r_{p} e^{i \Theta_{\ell}^{-}(k)}\right) \quad(p \in\{k-1, k\}, 0 \leq \ell \leq k+1)
$$

in $\partial \mathcal{A}_{k} \cap \mathcal{U}$ so that whenever $z \in S\left(r_{k}\right)$ has representations $z=r_{k} e^{i \Theta_{\ell}^{+}(k)}$ and $z=r_{k} e^{i \Theta_{\ell^{\prime}}^{-}(k+1)}$ from (11), then

$$
U\left(r_{k} e^{i \Theta_{\ell}^{+}(k)}\right)=U\left(r_{k} e^{i \Theta_{\ell^{\prime}}^{-}(k+1)}\right),
$$

thus defining $U$ unambiguously on $\Gamma^{0} \cap\left(S\left(r_{k}\right) \cap \mathcal{U}\right)$.

These boundary values (13) will be made explicit in $\S 5$, (59)-(61), and depend only on the data $A$ (the analytic set) and $\psi$ (see (1), (2) and Theorem 1). This means that we may choose $\left\{\Lambda_{k}\right\} \uparrow \infty$ depending only on data $A$ and $\psi$, and arrange ab initio that

$$
\begin{aligned}
& \max _{\ell}\left|U\left(r_{k} e^{i \Theta_{\ell}}\right)\right|<\frac{7}{k} L\left(r_{k}\right) \quad(k \geq 1), \\
& \max _{\ell}\left|U\left(r_{k} e^{i \Theta_{\ell}\left(r_{k}\right)}\right)-U\left(r_{k-1} e^{i \Theta_{\ell}\left(r_{k-1}\right)}\right)\right|<\Lambda_{k}, \\
& \frac{\Lambda_{k}}{\log \left(r_{k} / r_{k-1}\right)} \searrow 0, \quad(k \rightarrow \infty)
\end{aligned}
$$

if the ratios $r_{k} / r_{k-1}(k \geq 1)$ are chosen large enough.

To extend $U$ to $\mathcal{A}_{k}$ given its boundary values on $\Gamma^{0} \cap\left(\mathcal{A}_{k} \cap \mathcal{U}\right)$, for each $r \in$ $\left(r_{k-1}, r_{k}\right)$ set $\Theta_{0}(r)=0, \Theta_{k+1}(r)=\pi$, and if $1 \leq \ell \leq k$, select arguments $\Theta_{\ell}(r)$ with $\Theta_{\ell}(r)<\Theta_{\ell+1}(r)$, so that as $r \downarrow r_{k-1}, \Theta_{\ell}(r) \rightarrow \Theta_{\ell}^{-}(k)$ and as $r \uparrow r_{k}, \Theta_{\ell}(r) \rightarrow$ $\Theta_{\ell}^{+}(k)$, while uniformly in $\ell$

$$
r\left|\Theta_{\ell}^{\prime}(r)\right|+r^{2}\left|\Theta_{\ell}^{\prime \prime}(r)\right|<2 L\left(r_{k}\right)^{-7 / 6} \quad\left(r_{k-1} \leq r \leq r_{k}\right),
$$

so that $\Theta_{\ell}(r)$ is continuous at $r \in\left[r_{k-1}, r_{k}\right]$. The estimate (15) can be guaranteed if the ratios $r_{k} / r_{k-1}(k \geq 1)$ are sufficiently large. Then, (recall (13)), we define $U$ 
on each $\left\{r e^{i \Theta_{\ell}(r)}, r_{k-1}<r<r_{k}\right\}$ as

$$
\begin{aligned}
U\left(r e^{i \Theta_{\ell}(r)}\right) & =U\left(r_{k-1} e^{i \Theta_{\ell}\left(r_{k-1}\right)}\right) \\
& +\frac{\log \left(r / r_{k-1}\right)}{\log \left(r_{k} / r_{k-1}\right)}\left(U\left(r_{k} e^{i \Theta_{\ell}\left(r_{k}\right)}\right)-U\left(r_{k-1} e^{i \Theta_{\ell}\left(r_{k-1}\right)}\right)\right),
\end{aligned}
$$

and use (3), (4) to extend $U$ to all of $\mathcal{A}_{k}$ (note from (12) that $U$ is continuous). We have already set $z_{0}=i r_{0}=r_{0} e^{i \Theta_{1}^{-}(1)}$, now viewing $z_{0}$ as a point of $\Gamma^{0} \cap \partial \mathcal{A}_{1}$.

As noted in $\S 2.1,(3)$ also yields functions $\Psi_{\ell}(r), r_{k-1} \leq r \leq r_{k}, 1 \leq \ell \leq k+2$ with $U\left(r e^{i \Psi_{\ell}(r)}\right)$ a local maximum in each $S(r) \cap \mathcal{U}$.

2.4. On $\Delta U$. Further progress depends on analyzing the charge $\Delta U$.

Lemma 1. Let $U$ be continuous in $\mathbb{C}$ and $|\partial U / \partial \theta|=L(r)$ when $z \in\left(S(r) \cap \mathcal{A}_{k}\right) \backslash \Gamma^{\sharp}$ for $k \geq 0$.

Let the arcs of each $\mathcal{A}_{k} \cap \Gamma^{\sharp}:=\left\{r e^{i \Theta_{\ell}(r)}, r e^{i \Psi_{\ell}(r)}\right\}, r_{k-1} \leq r \leq r_{k}$ satisfy (15) and $U$ be assigned on $\mathcal{A}_{k}$ using its values on $\Gamma^{0} \cap\left(\partial \mathcal{A}_{k} \cap \mathcal{U}\right)$ as in (3), (4), (13) and (16). Finally, let $\delta_{a}(\theta)$ be the Dirac function (point mass) supported at $\theta=a$.

Then if $k \geq 0$ and $z \in \mathcal{A}_{k}, \Delta U(z)$ may be represented

$$
\Delta U\left(r e^{i \theta}\right)=2 r^{-2} L(r)\left[\sum_{\theta_{0} \in \Gamma^{0}} \delta_{\theta_{0}}(\theta)-\sum_{\theta^{*} \in \Gamma^{*}} \delta_{\theta^{*}}(\theta)\right]+H(r, \theta)+H_{\mathcal{A}}(r, \theta) .
$$

In (17), $H$ is differentiable,

$$
r^{2}|H(r, \theta)| \leq C^{\prime}, \quad \lim _{r \rightarrow \infty} \sup _{\theta}\left\{r^{2}|H(r, \theta)|\right\}=0 .
$$

In addition, $H_{\mathcal{A}}(r, \theta)$ has support on $\cup \partial \mathcal{A}_{k}$, with $H_{\mathcal{A}}\left(r_{k}, \theta\right)=\varepsilon_{k}(\theta)$ its density with respect to the Lebesgue measure on $S\left(r_{k}\right)$, where

$$
\sup _{r_{k} e^{i \theta} \in S\left(r_{k}\right)}\left|\varepsilon_{k}(\theta)\right|=o(1) \quad(k \rightarrow \infty) .
$$

Proof. Since $U=0$ for $\{|z|<1\}$, (3) and (4) show that $\Delta U=0$ on the real axis (including at $z=0$ ). When $z \in \Gamma^{\sharp} \cap \mathcal{A}_{k}$, (5) produces the bracketed term on the right side of (17) which is the main contribution to $\Delta U$.

We first study the primary error term $H(r, \theta)$ in (17). Thus suppose that $z \in(\mathcal{U} \cap$ $\left.\stackrel{\circ}{\mathcal{A}}_{k}\right) \backslash \Gamma^{\sharp}$. As in $\S 2.1$, we compute using polar coordinates. Assume for concreteness that

$$
U\left(r e^{i \theta}\right)=U\left(r e^{i \Theta_{\ell}(r)}\right)+L(r)\left(\theta-\Theta_{\ell}(r)\right),
$$

(see (3)). Then $\Delta U\left(r e^{i \Theta_{\ell}(r)}\right)=0$ (recall (16)). Hence

$$
\begin{aligned}
& \Delta U\left(r e^{i \Theta_{\ell}(r)}\right)=L^{\prime \prime}(r)\left(\theta-\Theta_{\ell}(r)\right)-2 L^{\prime}(r) \Theta_{\ell}^{\prime}(r)-L(r) \Theta_{\ell}^{\prime \prime}(r) \\
& +r^{-1}\left[L^{\prime}(r)\left(\theta-\Theta_{\ell}(r)\right)-L(r) \Theta_{\ell}^{\prime}(r)\right]:=H(r, \theta) .
\end{aligned}
$$

(with a change of sign when $U\left(r e^{i \theta}\right)=U\left(r e^{i \Theta_{\ell}(r)}\right)-L(r)\left(\theta-\Theta_{\ell}(r)\right)$ ). Moreover, since $\left|\theta-\Theta_{\ell}(r)\right|<\pi$, we obtain (18) for $z \in \mathcal{U}$ by estimating $r^{2} H(r, \theta)$ using (7), (9) and (15). For example, if $r_{k-1}<r<r_{k}$ then $r^{2}\left(r^{-1} L(r)\left|\Theta_{\ell}^{\prime}(r)\right|\right)=L(r) r\left|\Theta_{\ell}^{\prime}(r)\right| \leq$ $L\left(r_{k}\right)^{-1 / 6}=o(1)$ and $r^{2} L^{\prime}(r) \Theta_{\ell}^{\prime}(r)=O\left(r L^{\prime}(r) L\left(r_{k}^{-7 / 6}\right)\right)=o(1)$ as $k \rightarrow \infty$. The second item of (18) follows again from (4), (7), (9) and (15). 
It remains to consider (19), so let $\mathcal{A}(k, \eta)=\left\{z:|| z\left|-r_{k}\right|<\eta\right\}$. Let $\rho<\eta / 2$ and for $z \in \mathcal{A}(k, \eta / 2)$ we compute the Laplacian using the formula

$$
\Delta U(z)=\varliminf_{\rho \rightarrow 0} \frac{1}{4 \pi \rho^{2}}\left[\frac{1}{2 \pi} \int_{0}^{2 \pi}\left(U\left(z+\rho e^{i \phi}\right)-U(z)\right) d \phi\right]:=\varliminf_{\rho \rightarrow 0} \frac{1}{4 \pi \rho^{2}} U_{\rho}(z),
$$

with (for the moment) $z \notin \Gamma^{\sharp}$. Since $U_{\rho}$ is a Lipschitz function for each $\rho>0$, (19) is a consequence of the estimate (uniform in $\rho$ for $z \in \mathcal{A}(k, \eta / 2)$ )

$$
\varliminf_{\rho \rightarrow 0}\left|\rho^{-2} \int_{\mathcal{A}(k, \eta)} U_{\rho}(z) r d r d \theta\right|=o(1), \quad(k \rightarrow \infty) .
$$

To show (21), we follow Baernstein [2] and write, for $z=r e^{i \theta},\left|z+\rho e^{i \phi}\right|=$ $r(\phi), \arg \left(z+\rho e^{i \phi}\right)=\alpha(\phi)$, so that $r e^{i \theta}+\rho e^{i(\theta+\phi)}=r(\phi) e^{i(\theta+\alpha(\phi))}$. Since $z_{0} \notin$ $\Gamma^{\sharp}$, we may assume that $U$ is given by $(20)$ near $z$. Note that $r(\phi)=r(-\phi)$, $(\alpha(\phi)+\alpha(-\phi))=0$, so on collecting $\phi,-\phi$, the integrand in this computation of $\Delta U$ becomes

$$
\begin{aligned}
U\left(r(\phi) e^{i(\theta+\alpha(\phi))}\right)+U\left(r(\phi) e^{i(\theta-\alpha(\phi))}\right)-2 U\left(r e^{i \theta}\right) \\
=2\left[U\left(r(\phi) e^{i \Theta_{\ell}(r(\phi))}\right)+L(r(\phi))\left(\theta+((\alpha(\phi)+\alpha(-\phi)) / 2)-\Theta_{\ell}(r(\phi))\right)\right. \\
\left.\quad-U\left(r e^{i \theta}\right)\right] \\
=2\left[\left(U\left(r(\phi) e^{i \Theta_{\ell}(r(\phi))}\right)-U\left(r e^{i \Theta_{\ell}(r)}\right)\right)+\left(U\left(r e^{i \Theta_{\ell}(r)}\right)-U\left(r e^{i \theta}\right)\right)\right. \\
\quad+\left(L(r(\phi))\left(\theta-\Theta_{\ell}(r(\phi))\right)\right]:=I_{1}+I_{2}+I_{3} .
\end{aligned}
$$

For concreteness take $r=|z|>r_{k}, z \in \mathcal{A}(k, \eta / 2)$. Then if $|\phi|<\pi / 2$, both $z$ and $r(\phi) e^{i(\theta+\alpha(\phi))}$ are in $\mathcal{A}_{k+1}$, and so (16) applies. The main contribution to (22) will be from $I_{1}$. Our assumptions on $z$ and $\alpha(\phi)$ with (16) imply that

$$
\begin{aligned}
\frac{1}{2}\left|I_{1}\right| & =\left|U\left(r(\phi) e^{i \Theta_{\ell}(r(\phi))}\right)-U\left(r e^{i \Theta_{\ell}(r)}\right)\right| \\
& =\left|\frac{\log (r(\phi) / r)}{\log \left(r_{k+1} / r_{k}\right)}\right|\left(U\left(r_{k+1} e^{i \Theta_{\ell}\left(r_{k+1}\right)}\right)-U\left(r_{k} e^{i \Theta_{\ell}\left(r_{k}\right)}\right)\right) .
\end{aligned}
$$

Hence by (14)

$$
\left|I_{1}\right| \leq 2 \frac{\Lambda_{k+1}}{\log \left(r_{k+1} / r_{k}\right)} \log (r(\phi) / r) \leq C \frac{\Lambda_{k+1}}{\log \left(r_{k+1} / r_{k}\right)} \cdot \frac{\rho}{r_{k}},
$$

where we have used that $z \in \mathcal{A}(k, \eta / 2), r(\phi)>r_{k}$ and $|r-r(\phi)|<\rho$ to obtain the last inequality. If $|\phi-\pi|<\pi / 2$ and $r_{k}+\rho<r$, the same estimate holds for $I_{1}$.

When $r_{k}<r<r_{k}+\rho$, the point $r(\phi) e^{i(\theta+\alpha(\phi))}$ will be either in $\mathcal{A}_{k}$ or $\mathcal{A}_{k-1}$. In the former case, we repeat what was just done. Otherwise, the index $\ell$ may change in the sense that $U\left(r(\phi) e^{i(\theta+\alpha(\phi))}\right)$ may be given by (20) using $\Theta_{\ell^{\prime}}(r(\phi))$ with (perhaps) $\ell^{\prime} \neq \ell$ if $r(\phi) e^{i(\theta+\alpha(\phi))} \in \mathcal{A}_{k-1}$. However, since

$$
\begin{aligned}
& U\left(r(\phi) e^{i \Theta_{\ell^{\prime}}(r(\phi))}\right)-U\left(r e^{i \Theta_{\ell}(r)}\right)=\left(U\left(r(\phi) e^{i \Theta_{\ell^{\prime}}(r(\phi))}\right)-U\left(r_{k} e^{i \Theta_{\ell}\left(r_{k}\right)}\right)\right) \\
& +\left(U\left(r_{k} e^{i \Theta_{\ell}\left(r_{k}\right)}\right)-U\left(r e^{i \Theta_{\ell}(r)}\right)\right),
\end{aligned}
$$

we still may arrange that

$\frac{1}{2}\left|I_{1}\right| \leq \frac{\Lambda_{k}}{\log \left(r_{k} / r_{k-1}\right)} \log \left(r_{k} / r(\phi)\right)+\frac{\Lambda_{k+1}}{\log \left(r_{k+1} / r_{k}\right)} \log \left(r / r_{k}\right) \leq C \frac{\Lambda_{k}}{\log \left(r_{k} / r_{k-1}\right)} \cdot \frac{\rho}{r_{k}}$, since $r(\phi)<r_{k}<r, r-r(\phi)<\rho$, and (14). 
Analogous estimates apply when $r_{k}-\eta<r<r_{k}$. We integrate this over $\mathcal{A}(k, \eta)$, whose area is $O\left(r_{k} \eta\right)$, and recall that $\rho<\eta / 2$. Hence

$$
\int_{\mathcal{A}(k, \eta)} \rho^{-2}\left|I_{1}\right| r d r d \theta \leq C \frac{\Lambda_{k}}{\log \left(r_{k} / r_{k-1}\right)} \cdot \frac{\eta}{\rho} .
$$

As for $I_{2}$ and $I_{3}$ from (22), (16) and (20) show that

$$
\begin{aligned}
& \frac{1}{2}\left(I_{2}+I_{3}\right)=U\left(r_{k} e^{i \Theta_{\ell}\left(r_{k}\right)}\right)-U\left(r e^{i \theta}\right)+L(r(\phi))\left(\theta-\Theta_{\ell}(r(\phi))\right) \\
& =-L(r)\left(\theta-\Theta_{\ell}(r)\right)+L(r(\phi))\left(\theta-\Theta_{\ell}(r(\phi))\right. \\
& =(L(r(\phi))-L(r))\left(\theta-\Theta_{\ell}(r(\phi))+L(r)\left(\Theta_{\ell}(r)-\Theta_{\ell}(r(\phi)) .\right.\right.
\end{aligned}
$$

The estimates of the first derivatives of $L(r), \Theta_{\ell}(r)$ from (7) and (15) are exploited in a manner similar to that used in estimating $I_{1}$, and so

$$
\int_{\mathcal{A}(k, \eta)} \rho^{-2}\left(\left|I_{2}\right|+\left|I_{3}\right|\right) r d r d \theta=\frac{\eta}{\rho} o(1), \quad(k \rightarrow \infty)
$$

yielding the first estimate of (19).

(This argument also shows that the contribution to (19) from the $O(k)$ points of $S(r) \cap \Gamma^{\sharp}$ can also be absorbed in this type of estimate.)

2.5. Refined properties of $U$. That $w=a$ be an asymptotic value of $f$ on a curve $\gamma$ requires information for all large $r=|z| \in \gamma$, and one needs equally precise information on a significant portion of the plane to ensure that if $a \notin A^{*}$, then $a$ cannot be an asymptotic value. To surmount problems arising from the inevitable exceptional sets which arise in approximation theory, we impose conditions on the functions $\left\{\Theta_{\ell}\right\}$ of (15). Some of these might be weakened or perhaps avoided at the price of complicating the proofs of the key Theorem 3 (§3.6) and Lemma 8.

For each $k \geq 1$, define $\rho_{k-1}$ by

$$
\log \left(\rho_{k-1} / r_{k-1}\right)=L^{1 / 4}\left(r_{k-1}\right),
$$

so that $S\left(\rho_{k-1}\right) \subset \mathcal{A}_{k}$, while (9) shows that $\rho_{k-1} / r_{k-1}=o\left(r_{k} / r_{k-1}\right)$. Note from the first term in (7) and (23) that $L\left(r_{k-1}\right) \asymp L\left(\rho_{k-1}\right)$ :

$$
L\left(r_{k-1}\right) \leq L\left(\rho_{k-1}\right)=L\left(r_{k-1}\right)+o(1) \log \left(\rho_{k-1} / r_{k-1}\right)=(1+o(1)) L\left(r_{k-1}\right) .
$$

In addition, we define $r_{k}^{\prime}, r_{k}^{\prime \prime}$, where $\rho_{k-1}<r_{k}^{\prime}<r_{k}^{\prime \prime}<r_{k}$ so that

$$
\rho_{k-1}=o\left(r_{k}^{\prime}\right) ; \quad r_{k}^{\prime}=o\left(r_{k}^{\prime \prime}\right) \quad r_{k}^{\prime \prime}=o\left(r_{k}\right) .
$$

In particular, let

$$
\log \left(r_{k}^{\prime} / \rho_{k-1}\right)=L^{1 / 3}\left(r_{k-1}\right)
$$

and set

$$
\mathcal{K}=\bigcup_{k}\left\{r_{k}^{\prime} \leq|z| \leq r_{k}^{\prime \prime}\right\}
$$

the core of $\cup \mathcal{A}_{k}$.

Note from (10) that $U$ is known on $\mathcal{A}_{0}$, and by (11) and (4)

$$
\operatorname{card}\left(S\left(r_{k}\right) \cap \mathcal{A}_{k+1} \cap \Gamma^{0}\right)=\operatorname{card}\left(S\left(r_{k}\right) \cap \overline{\mathcal{A}_{k}} \cap \Gamma^{0}\right)+2 \quad(k \geq 1) .
$$

Thus the condition (12) will be satisfied by requiring that two $\operatorname{arcs}\left\{\Theta_{\ell}(r)\right\}$ in $\mathcal{A}_{k}$ emerge from a common point of $S\left(r_{k}\right) \cap \mathcal{U}(k \geq 2)$. Hence $\left\{\Gamma^{0}\right\}$ undergoes a bifurcation on $S\left(r_{k}\right) \cap \mathcal{U}$ (in turn creating another bifurcation of $\Gamma^{*}$ on $S\left(r_{k}\right) \backslash \mathcal{U}$ ). The bifurcation points $\pm z_{k} \in S\left(r_{k}\right)$ are called nodes of $\Gamma^{0}$, so that $\Gamma^{0} \cap \mathcal{U}$ is a dyadic tree. In $\S 5$ we identify the branches of $\Gamma^{0} \cap \mathcal{U}$ in terms of the nodes $\left\{z_{k}\right\}$ 
through which they pass. As an $\operatorname{arc} \gamma \subset \Gamma^{0}$ recedes, its index $\Theta_{\ell}$ relative to $\mathcal{A}_{k}$ will also depend on $k$ (see Figure 2 which represents $\Gamma^{\sharp}$ in $\mathcal{U} \cap\left\{r_{4}<|z|<r_{8}\right\}$ ). On the outer boundary $S\left(r_{k}\right)$ of each $\partial \mathcal{A}_{k} \cap \mathcal{U}$, the arguments $\Theta_{\ell}^{+}$in (11) are chosen to have the form

$$
\Theta_{\ell}^{+}(k)=\frac{\ell}{k+1} \pi \quad(0 \leq \ell \leq k+1) .
$$

We then locate the bifurcation node $z_{k} \in S\left(r_{k}\right)$, now viewed as the inner boundary of $\mathcal{A}_{k+1} \cap \mathcal{U}$ so that if $k=2^{n}+p, 0 \leq p \leq 2^{n}-1$, then

$$
\begin{array}{ll}
\Theta_{\ell}^{-}(k)=\frac{\ell}{k+1} \pi & \text { for } 1 \leq \ell \leq 2 p+1, \\
\Theta_{\ell}^{-}(k)=\frac{\ell-1}{k+1} \pi & \text { for } 2 p+2 \leq \ell \leq k+2 ;
\end{array}
$$

thus $\Theta_{2 p+1}^{-}(k)=\Theta_{2 p+2}^{-}(k)$, guaranteeing (11) and (12). We then use (13)-(16) with (3) and (4) to extend $U$ to $\mathbb{C} \cap\left\{|z|>r_{0}\right\}=\cup_{k \geq 1} \mathcal{A}_{k}$.

In Figure 2 (not to scale) $\Gamma^{0}$ is indicated with solid lines and $\Gamma^{*}$ with dashed lines. The symbols $\Theta_{\ell}(k)$ are labeling the nodes with argument $\Theta_{\ell}(k)$.

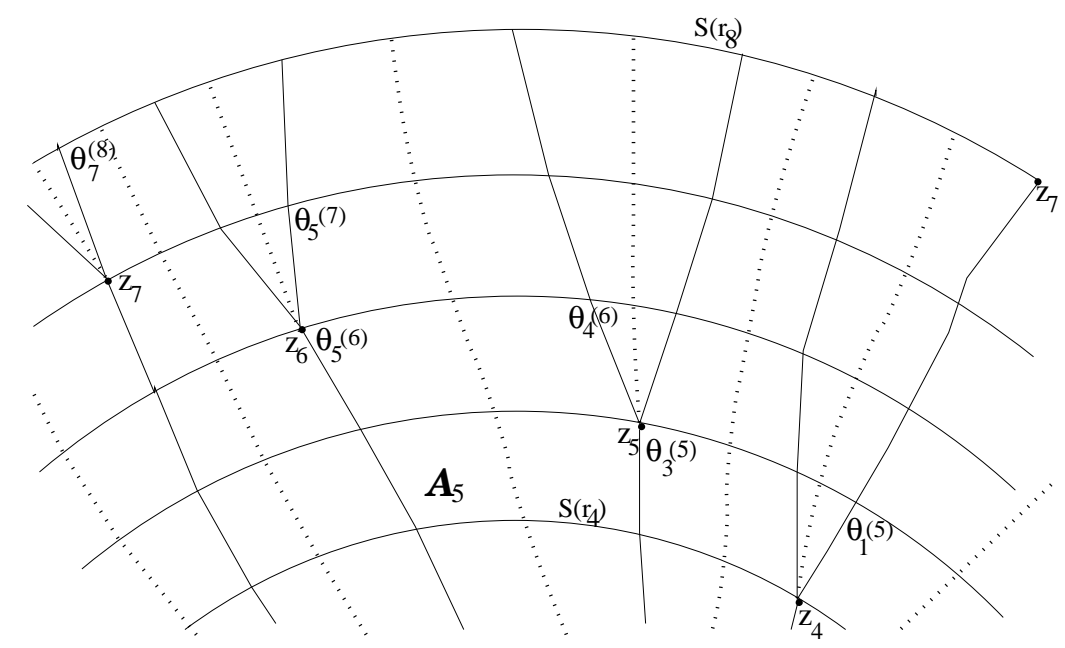

Figure 2. The trace of $\Gamma^{\sharp}$.

With $\rho_{k-1}$ from (23), construct $\Gamma^{0} \cap \mathcal{A}_{k}$ with initial conditions (26) and (consistent with (7))

$$
\begin{array}{ll}
r \Theta_{2 p+1}^{\prime}(r)=-r \Theta_{2 p+2}^{\prime}(r)=L^{-3 / 4}\left(r_{k-1}\right) & \left(r_{k-1} \leq r \leq \rho_{k-1}\right) \\
\text { and if } \ell \neq 2 p+1,2 p+2 & \\
\Theta_{\ell}^{\prime}=0 & \left(r_{k-1}<r<\rho_{k-1}\right)
\end{array}
$$

(where' ${ }^{\prime}$ is differentiation with respect to $r$ ) as illustrated in Figure 2. 
It follows using (27), (23) and the first condition of (9) that

$\left|\Theta_{2 p+1}\left(\rho_{k-1}\right)-\Theta_{2 p+1}\left(r_{k-1}\right)\right|=L^{-3 / 4}\left(r_{k-1}\right) \log \left(\frac{\rho_{k-1}}{r_{k-1}}\right)=(1+o(1)) L^{-1 / 2}\left(\rho_{k-1}\right)$.

Moreover, since (25) and the second property of (7) guarantee that on $S\left(r_{k}\right)$ distinct points of $\Gamma^{0}$ have angular separation

$$
\pi /(k+1)>\pi L\left(r_{k}\right)^{-1 / 3}>\pi(1+o(1)) L\left(\rho_{k}\right)^{-1 / 3},
$$

the second line of (27) will show that if $r e^{i \tau(r)}, r e^{i \tau^{\prime}(r)} \in \Gamma^{0} \cap(S(r) \cap \mathcal{U})$ then

$$
\left|\tau(r)-\tau^{\prime}(r)\right|>L^{-2 / 9}(r) \quad\left(\rho_{k}<r<r_{k+1}\right) .
$$

On recalling (3) and (15), it is not difficult to see that (28) then holds as well on $\Gamma^{\sharp} \cap S(r)$ when $r_{k} \leq r \leq r_{k+1}$ except for $\Theta_{2 p}$ and $\Theta_{2 p+1}$.

Finally, in the core $\left\{r_{k}^{\prime}<|z|<r_{k}^{\prime \prime}\right\}$ of each $\mathcal{A}_{k}$ (recall (24)) we require that

$$
\Theta_{\ell}^{\prime}(r)=0 \quad\left(1 \leq \ell \leq k+1, r_{k}^{\prime}<r<r_{k}\right)
$$

\section{Approximation by a Meromorphic FUnCtion}

The idea that the behaviour of a general $\delta$-subharmonic function $U$ can be captured by another of the special form $\log |g|$ with $g$ meromorphic goes back several decades (a survey is in [6], additional interesting references are [18], [12], [9], among others).

In our situation the error $|\log | g(z)|-U(z)|$ must be carefully controlled which is formalized in the next theorem.

Theorem 2. Let $L(r)$ be a function which satisfies (7), let the system $\left\{\mathcal{A}_{k}\right\}_{k \geq 0}$ satisfy (6) and (9), where (increasing each of the ratios $r_{k+1} / r_{k}$ if necessary)

$$
\int_{r_{k}}^{r_{k+1}} L(t) t^{-1} d t \text { is an integer, }
$$

and let $U$ be constructed relative to the system $\left\{\mathcal{A}_{k}\right\}$ so that $U(z)=0$ for $z$ real and $z \in B(0,1), U$ is assigned to the network $\Gamma^{0} \cap \mathcal{U}$ as in (16) so that $U$ is continuous relative to $\Gamma^{0} \cap \mathcal{U}$, and then extended to each $\mathcal{A}_{k}$ using (3) and (4).

Then there is a meromorphic function $g(z)$ and an absolute constant $C_{0}>0$ such that if

$$
E=\bigcup B\left(\zeta_{p},\left|\zeta_{p}\right| / 10 L\left(\left|\zeta_{p}\right|\right)\right)
$$

with $\left\{\zeta_{p}\right\}$ the zeros and poles of $g$, then

(a) meas $(E \cap S(r))=o(r) \quad(r \rightarrow \infty)$;

(b) if $z \notin E$ then $|\log | g(z)|-U(z)|<C_{0}$;

(c) if $E^{\prime}$ is a component which contains one point-mass $\zeta_{p}$, then for sufficiently large $r$

$$
\begin{aligned}
& \log |g(z)| \leq U(z)+C_{0}, \quad z \in E^{\prime}, \zeta_{p} \text { zero of } g \\
& \log |g(z)| \geq U(z)-C_{0}, \quad z \in E^{\prime}, \zeta_{p} \text { pole of } g .
\end{aligned}
$$

The behavior of $g$ on components $E^{\prime}$ of $E$ which are not disks is more delicate, and requires the additional structure introduced in $\S 2.5$ : see $\S 3.6$. 
Results such as Theorem 2 depend on analysis of the (signed) measure $\Delta U$, so we prove Theorem 2 as formulated in Theorem $2^{\prime}$. Write $\Delta U$ from Lemma 1 as

$$
\Delta U=\mu-\mu^{*}+\mu_{e},
$$

with support on $\{|z| \geq 1\}$, where where $\mu \geq 0$ is supported on $\Gamma^{0}, \mu^{*} \geq 0$ on $\Gamma^{*}$ and $d \mu_{e}(z)=H(r, \theta) r d r d \theta+H_{\mathcal{A}}(r, \theta) d \theta$, with $H_{\mathcal{A}}$ supported on $\cup_{k} \mathcal{A}_{k}$. Since $g$ is meromorphic, $\Delta \log |g|$ is a network of unit masses, so that $\Delta \log |g|=\sigma-\sigma^{*}+\sigma_{e}$, each summand corresponding to a term of $\Delta U$.

By construction, each component of $\Gamma^{\sharp} \cap \mathcal{A}_{k}$ is an arc joining the boundary components of $\mathcal{A}_{k}$, relative to which $\Delta U$ becomes one of the terms in the first two summands of (17). Using (30), each component $\gamma$ is the union of mutually disjoint $\operatorname{arcs}\{J\}$ of 'measure' \pm 1 . Since $L$ vanishes on $[0,1], \mu+\mu^{*}+\mu_{e}$ vanishes on $B(0,1)$, and (4) shows that

$$
\mu(S)=\mu^{*}(-S) \text { for all measureable sets } S .
$$

Let $J \subset \Gamma^{0}$ such that $\mu(J)=1$ and recall that the density $d \mu$ is given by (17), that is $d \mu \sim(2 L(r) / r) d r$. Then conditions (7), (9) on the growth of $L(r)$ and (15), (27) and (29) (that show that $J$ is almost a radial segment) imply that

$$
J \subset\left\{r \leq|z| \leq r\left(1+\frac{1}{L(r)}\right)\right\} \quad \text { and } \quad \frac{r}{3 L(r)} \leq|J| \leq \frac{3 r}{2 L(r)},
$$

for some $r=r(J)>r_{0}$. The same estimates hold when $J \in \Gamma^{*}$ with $\mu^{*}(J)=1$.

3.1. A reformulation. The logarithmic potential of a signed measure $\Sigma$ of compact support is defined as

$$
P(z, \Sigma)=\int_{\mathbb{C}} \log |1-z / \zeta| d \Sigma(\zeta)
$$

which is $\delta$-subharmonic (subharmonic when $\Sigma \geq 0$ ). Our measures do not have compact support which means the formula has to be carefully interpreted, which we achieve by appropriate pairing of measures. We recall measures $\mu, \mu^{*}$ and (the signed measure) $\mu_{e}$ in (32) and follow a standard procedure (c.f. [6]) to "atomize" the first two measures obtaining $\sigma$ and $\sigma^{*}$. This leads to the expressions:

$$
G(z):=U(z)+V(z)
$$

where

$$
\begin{aligned}
& V(z):=V_{\Gamma^{\sharp}}(z)+V_{e}(z), \\
& V_{\Gamma^{\sharp}}(z):=P(z, \sigma-\mu)-P\left(z, \sigma^{*}-\mu^{*}\right), \\
& V_{e}(z):=-P\left(z, \mu_{e}\right) .
\end{aligned}
$$

We will show directly that $V$ is well-defined: each of the two summands defining $V_{\Gamma^{\sharp}}$ converges, while not only does $V_{e}$ converge, but $V_{e}(z)=o(1)$. Thus there is a meromorphic function in the plane $g$ with $G(z)=\log |g(z)|$. Our estimates will show that for most $z,|G(z)|$ is small, where we apply techniques such as in [12], [14] or [6].

Recall that $\Gamma^{\sharp} \cap \mathcal{A}_{k}$ is a union of intervals $J$ and $J^{*}$ so that $|\mu(J)|=1$ and $\mu^{*}\left(J^{*}\right)=1$. To construct $\sigma$ we consider an interval $J \subset \gamma \subset \Gamma^{0} \cap \mathcal{A}_{k}$, with $\mu(J)=1$. Following [18] we place the associated point mass at its centroid $\zeta_{J}$,

$$
\int_{J}\left(\zeta-\zeta_{J}\right) d \mu(\zeta)=0
$$


so that $\delta_{\zeta_{J}}$ is a term of $\sigma$. The same principle yields $\left\{\zeta_{J^{*}}\right\} \subset \Gamma^{*} \cap \mathcal{A}_{k}$ using $\mu^{*}$. Notice from (33) and (35) that the $\left\{\zeta_{J}, \zeta_{J^{*}}\right\}$ may be put into correspondence with

$$
\zeta_{J^{*}}=-\zeta_{J}, \quad \text { when } J^{*}=-J .
$$

The measure $\mu_{e}$ does not need atomization since it is very small. The analysis of $V_{e}$ is presented in $\S 3.3$.

We thus restate the assertions of Theorem 2 in terms of these approximating measures. To simplify notation, we often let $I$ be a generic choice of $J$ or $J^{*}$. In Theorem $2^{\prime}$, the centers $\left\{\zeta_{p}\right\}$ of $(31)$ are the $\left\{\zeta_{J}, \zeta_{J^{*}}\right\}$. Assertion $(a)$ in these theorems is equivalent, but assertions $(b)$ and $(d)$ of Theorem $2^{\prime}$ correspond to $(b)$ in Theorem 2, and $(c)$ and $(d)$ in Theorem $2^{\prime}$ to $(c)$ in Theorem 2.

Theorem 2'. Under the assumptions of Theorem 2, let $\left\{\zeta_{I}\right\}$ be the centroids of the intervals $I$, where $I \in \Gamma^{0}$ or $\Gamma^{*}$. Let $E$ be as in (31) and $\zeta_{p}=\zeta_{I}$. Then

(a) $\operatorname{meas}(E \cap S(r))=o(r)$, as $r \rightarrow \infty$,

(b) $\left|V_{\Gamma^{\sharp}}(z)\right|<C_{0} \quad(z \notin E)$,

(c) if $z \in B\left(\zeta_{I},\left|\zeta_{I}\right| / 5 L\left(\left|\zeta_{I}\right|\right)\right)$ and $B\left(\zeta_{I},\left|\zeta_{I}\right| / 5 L\left(\left|\zeta_{I}\right|\right)\right)$ is a component of $E$, then

$$
\begin{aligned}
& V_{\Gamma^{\sharp}}(z) \leq C_{0}, \quad \text { if } \zeta_{I} \text { a zero of } g, \\
& V_{\Gamma^{\sharp}}(z) \geq-C_{0} \quad \text { if } \zeta_{I} \text { a pole of } g,
\end{aligned}
$$

(d) $\left|V_{e}(z)\right|=o(1) \quad(z \rightarrow \infty)$.

Note, since $L(r) \uparrow \infty$, that (28) and (34) imply that all balls $B\left(z_{p},\left|z_{p}\right| / 5 L\left(\left|z_{p}\right|\right) \subset\right.$ $\mathcal{K}$ (from $(24)$ ) are disjoint, and so (23) implies that $(d)$ holds in most of $\mathbb{C}$. The situation in $\mathbb{C} \backslash \mathcal{K}$ is settled in Theorem 3 in $\S 3.6$.

3.2. Proof of Theorem $\mathbf{2}^{\prime}(a)$. The description of $\Gamma^{\sharp}$ in $\S 2$ implies that the number of points in $S(r) \cap \Gamma^{\sharp}$ for $r \in \mathcal{A}_{k}$ is at most $4 k+2$, and the angular measure of each ball in $E$ is $O(1 / L(r))$. Thus the total angular measure of $E \cap S(r)$ for $r_{k} \leq r \leq r_{k+1}$ is $O(k / L(r))$, so (9) gives

$$
\operatorname{meas}(E \cap S(r))=O\left(r L^{-1+1 / 3}(r)\right)=o(r) \quad r \rightarrow \infty .
$$

3.3. Proof of Theorem $\mathbf{2}^{\prime}(d)$. It is simple to estimate $V_{e}$ from (3.1). That $\mu_{e}$ is uniformly small follows from (18) and the first of (19). Hence assertion $(d)$ follows from the next lemma.

Lemma 2. The function $V_{e}(z)$ satisfies

$$
\left|V_{e}(z)\right|=\left|\int_{\mathbb{C}} \log \right| 1-z / \zeta\left|d \mu_{e}(\zeta)\right|=o(1) \quad(|z| \rightarrow \infty) .
$$

Proof. First consider the contribution to $d \mu_{e}$ from $d \mu_{e}^{1}:=H(r, \theta) r d r d \theta$. Since (4) implies that $H(r, \theta)=-H(r, \theta+\pi)(0 \leq \theta<\pi)$.

$$
\int_{\mathbb{C}} \log |1-z / \zeta| d \mu_{e}^{1}(\zeta)=\int_{\mathbb{C}}(\log |1-z / \zeta|-\log |1+z / \zeta|) d\left(\mu_{e}^{1}\right)^{+}(\zeta),
$$


where $\left(\mu_{e}^{1}\right)^{+}(\zeta)$ is the positive part of $\mu_{e}^{1}(\zeta)$. Standard estimates then yield that

$$
\begin{aligned}
& |\log | \frac{1-z / \zeta}{1+z / \zeta}|| \leq C\left|\frac{z}{\zeta}\right|=C \frac{r}{|\zeta|} \quad(2 r<|\zeta|), \\
& |\log | \frac{1-z / \zeta}{1+z / \zeta}|| \leq C\left|\frac{\zeta}{z}\right|=C \frac{|\zeta|}{r} \quad(2|\zeta|<r) .
\end{aligned}
$$

By (18), given $\epsilon>0$ there exists $r_{\epsilon}$ with $r^{2} H<\epsilon$ for $r>r_{\epsilon}$. Then when $r>r_{\epsilon} / \epsilon$,

$$
\begin{gathered}
\int_{\{|\zeta|>2 r\}}|\log | \frac{1-z / \zeta}{1+z / \zeta}|| d\left(\mu_{e}^{1}\right)^{+}(\zeta)+\int_{\{|\zeta|<r / 2\}}|\log | \frac{1-z / \zeta}{1+z / \zeta}|| d\left(\mu_{e}^{1}\right)^{+}(\zeta) \\
\leq C \epsilon r \int_{2 r}^{\infty} \frac{1}{t^{2}} d t+\frac{C}{r} \int_{0}^{r(\epsilon)} d t+C \frac{\epsilon}{r} \int_{r(\epsilon)}^{r / 2} d t \leq C \epsilon .
\end{gathered}
$$

Now $d \mu_{e}$ is smooth and satisfies (18), and so

$$
\int_{\{|\log | \zeta / z||<\log 2\}} \log |1-z / \zeta| d\left(\mu_{e}^{1}\right)^{+}(\zeta)=o(1) \quad(r \rightarrow \infty) .
$$

Estimate (19) and the fact that the sequence $\left\{r_{k}\right\}_{k>0}$ is rapidly increasing give the same bound for the contribution to $d \mu_{e}$ from $H_{\mathcal{A}}(r, \theta)$, with $H_{\mathcal{A}}$ from Lemma 1.

3.4. Proof of Theorem $\mathbf{2}^{\prime}(b)$. Controlling $V_{\Gamma^{\sharp}}$ is more complicated and needs several lemmas. The first estimates a single term, with $z$ not too near the centroid, based on work from [7].

Lemma 3. Let $J \in \Gamma^{0}$ be an interval of $\mu$-measure one. Let $J^{*}=-J \in \Gamma^{*}$ and $\zeta_{J}$ and $\zeta_{J^{*}}$ the associated centroids as in (35). Denote by $\mathbf{J}$ the ordered pair $\mathbf{J}=\left(J, J^{*}\right)$ and define

$$
h_{\mathbf{J}}(z):=\int_{J} \log \left|\frac{1-z / \zeta_{J}}{1-z / \zeta}\right| d \mu(\zeta)-\int_{J^{*}} \log \left|\frac{1-z / \zeta_{J^{*}}}{1-z / \zeta}\right| d \mu^{*}(\zeta) .
$$

Then if

$$
d\left(z, \zeta_{J} \cup \zeta_{J^{*}}\right) \geq 3 \frac{\left|\zeta_{J}\right|}{L\left(\left|\zeta_{J}\right|\right)}
$$

there exists an absolute constant $C>0$ with

$$
\left|h_{\mathbf{J}}(z)\right| \leq C\left(\frac{|J|}{\left|z-\zeta_{J}\right| \wedge\left|z-\zeta_{J^{*}}\right|}\right)^{2} .
$$

Proof. Let $\mathcal{B}=B\left(\zeta_{J}, \delta / 2\right)$ be the smallest disk centered at $\zeta_{J}$ which contains $J$, so that by (34), $\delta \leq 2\left|\zeta_{J}\right| / L\left(\left|\zeta_{J}\right|\right)$. Then, by $(39), z \notin B\left(\zeta_{J}, \delta\right)$, so we expand the function $\log ((\zeta-z) /(\zeta+z))$ about $\zeta_{J}$, with remainder of second order. The first-order term drops out due to (35), and thus

$$
\begin{aligned}
\left|h_{\mathbf{J}}(z)\right| & =\left|\int_{J} \log \right| \frac{\zeta-z}{\zeta+z}|-\log | \frac{\zeta_{J}-z}{\zeta_{J}+z}|d \mu(\zeta)| \\
& \leq C \max _{\mathcal{B}}\left|\frac{1}{(\zeta+z)^{2}}-\frac{1}{(\zeta-z)^{2}}\right| \int_{J}\left|\zeta-\zeta_{J}\right|^{2} d \mu(\zeta) .
\end{aligned}
$$

However $\left|\zeta-\zeta_{J}\right| \leq|J|, \mu(J)=1$ and the factor with the max is comparable to $\left(\left|z-\zeta_{J}\right| \wedge\left|z-\zeta_{J *}\right|\right)^{-2}$. This proves the lemma. 
Lemma 3 leads to the main estimate.

Lemma 4. Let $z \in \mathbb{C}$ satisfy (39) for all intervals $J, J^{*}$ in $\Gamma^{\sharp}$ (so by (31) $z \notin E$ ), let $\mathbf{J}=\left(J, J^{*}\right)$ and, using the notation in (38), write

$$
V_{\Gamma^{\sharp}}(z)=\sum_{\mathbf{J}} h_{\mathbf{J}}(z) .
$$

Then there exists an absolute constant $C$ so that

$$
\left|V_{\Gamma^{\sharp}}(z)\right| \leq \sum_{\mathbf{J}}\left|h_{\mathbf{J}}(z)\right| \leq C .
$$

Proof. Since we are assuming (39) holds for all $J, J^{*}$, let $\mathbf{J}=\left(J, J^{*}\right)$ and apply Lemma 3 to each term in the sum. Given $r=|z|$, divide the sum into three groups: $\mathcal{I}_{1}$ contains the pairs of intervals that are in $B\left(r L^{-3}(r)\right), \mathcal{I}_{2}$ those pairs of intervals with null intersection with $B\left(r L^{3}(r)\right)$, and $\mathcal{I}_{3}$ the others.

The estimate for $\mathcal{I}_{1}$ follows routinely from grouping the pairs of intervals as in the proof of Lemma 3 and using (37) combined with (17), (36) and the fact (cf. (9)) that $O\left(L^{1 / 3}(r)\right)$ points of $\Gamma^{\sharp}$ meet each $S(r)$ :

$$
\begin{aligned}
& \sum_{\mathbf{J} \subset \mathcal{I}_{1}}\left|h_{\mathbf{J}}(z)\right|=\sum_{J \subset B\left(r L^{-3}(r)\right)}\left|\int_{J} \log \right| \frac{1-z / \zeta_{J}}{1+z / \zeta_{J}}|-\log | \frac{1-z / \zeta}{1+z / \zeta}|d \mu(\zeta)| \\
& \leq C \int_{0}^{2 r L^{-3}(r)} \frac{t}{r} L^{1 / 3}(t) \frac{L(t)}{t} d t<C L^{-8 / 3}(r)=o(1) \quad(r \rightarrow \infty) .
\end{aligned}
$$

Next, consider the pairs of intervals in $\mathcal{I}_{2}$, and choose $m \in \mathbb{N}$ with $2^{m} \leq L^{3}(r)<$ $2^{m+1}: m \sim C \log L^{3}(r)$. For $n \geq m$, (9) shows that the annulus $\mathcal{A}_{(n)}:=\left\{2^{n} r \leq\right.$ $\left.|\zeta|<2^{n+1} r\right\}$ has $O\left(L^{1 / 3}\left(2^{n} r\right)\right)$ arcs of $\Gamma^{\sharp}$ joining its boundary components, each arc of which is the union of $O\left(L\left(2^{n} L(r)\right)\right)$ intervals of unit $\mu$-mass. The first estimate (37) gives for each term

$$
\left|\int_{J} \log \right| \frac{\zeta-z}{\zeta+z}|-\log | \frac{\zeta_{J}-z}{\zeta_{J}+z}|d \mu(\zeta)| \leq C r \int_{J} \frac{1}{|\zeta|} d \mu(\zeta),
$$

$C>0$ an absolute constant and $J \subset\left\{z:|z|>r L^{3}(r)\right\}$. The essential condition (7) yields that

$$
L\left(2^{n} r\right)=L(r)+o(n) .
$$

Since $\mu(J)=1,(9)$ and $2^{m}>C L^{3}(r)$, we have

$$
\begin{aligned}
& \sum_{J \subset\left\{|z|>r L^{3}(r)\right\}} r \int_{J} \frac{1}{|\zeta|} d \mu(\zeta) \leq C \sum_{n \geq m} \frac{L^{1 / 3}\left(2^{n} r\right) L\left(2^{n} r\right)}{2^{n}}<C \frac{L^{4 / 3}\left(2^{m} r\right)}{2^{m}} \\
& \leq C \frac{(L(r)+o(m))^{4 / 3}}{2^{m}} \leq \frac{C}{L(r)}=o(1) \quad(r \rightarrow \infty)
\end{aligned}
$$

(the ratio of successive terms in the series is $\frac{1}{2}+o(1)$ ).

Consider now the pairs of intervals in $\mathcal{I}_{3}$. All these intervals intersect the annulus $\left\{r L^{-3}(r)<|\zeta|<r L^{3}(r)\right\}$, and (39) holds for each of them. These pairs of intervals are apportioned into two groups. Take as $\mathcal{I}_{3}^{\prime}$ those pairs such that both intervals are in the core $\mathcal{B}:=\{r / 2<|\zeta|<2 r\}$; those pairs remaining are in $\mathcal{I}_{3}^{*}$. 
First consider the contribution from $\mathcal{I}_{3}^{*}$, intervals in annuli $\mathcal{A}_{(n)}$ with $n \leq-1$ or $1 \leq n<C \log L^{3}(r)=C \log L(r)$. When $n<-1$ and $J \subset \mathcal{A}_{(n)}$, then $|J|<$ $C 2^{n} r / L\left(2^{n} r\right)$ and $\left|z-\zeta_{J}\right| \wedge\left|z-\zeta_{J^{*}}\right|>(1 / 2+o(1)) r>r / 4$, so that

$$
\frac{|J|}{\left|z-\zeta_{J}\right| \wedge\left|z-\zeta_{J^{*}}\right|} \leq \frac{C 2^{n} r}{r L\left(2^{n} r\right)}=\frac{C 2^{n}}{L\left(2^{n} r\right)} .
$$

There are $O\left(L\left(2^{n} r\right)\right)$ intervals $J$ on each component of $\Gamma^{\sharp} \cap \mathcal{A}_{(n)}$ with $n \leq-1$, and (9) again shows there are most $C L^{1 / 3}\left(2^{n} r\right)$ branches in $\mathcal{A}_{(n)}$. Thus

$$
\begin{array}{r}
\sum_{n<-1} \sum_{J \subset \mathcal{A}_{(n)}}\left(\frac{|J|}{\left|z-\zeta_{J}\right| \wedge\left|z-\zeta_{J^{*}}\right|}\right)^{2} \leq C \sum_{n<-1} \frac{2^{2 n}}{L\left(2^{n} r\right)} L^{1 / 3}\left(2^{n} r\right) \\
<C \sum_{n<-1} L^{-2 / 3}\left(2^{n} r\right) 2^{2 n}=o(1) \quad(r \rightarrow \infty) .
\end{array}
$$

When $1 \leq n \leq C \log L(r)$ and $J \subset \mathcal{A}_{(n)},\left|z-\zeta_{J}\right| \wedge\left|z-\zeta_{J^{*}}\right| \geq C 2^{n} r$, and so

$$
\frac{|J|}{\left|z-\zeta_{J}\right| \wedge\left|z-\zeta_{J^{*}}\right|} \leq \frac{C 2^{n} r}{2^{n} r L\left(2^{n} r\right)}=\frac{C}{L\left(2^{n} r\right)} .
$$

There are $O\left(L\left(2^{n} r\right)\right)$ intervals $J$ in each component of $\mathcal{A}_{(n)} \cap \Gamma^{\sharp}$ and $O\left(L^{1 / 3}\left(2^{n} r\right)\right)$ such components with $n<C \log L(r)$. Hence

$$
\begin{array}{r}
\sum_{n=1}^{C \log L(r)} \sum_{J \subset \mathcal{A}_{(n)}}\left(\frac{|J|}{\left|z-\zeta_{J}\right| \wedge\left|z-\zeta_{J *}\right|}\right)^{2} \leq C \sum_{n=1}^{C \log L(r)} \frac{L^{1 / 3}\left(2^{n} r\right)}{L\left(2^{n} r\right)} \\
\leq C \frac{\log L(r)}{L^{2 / 3}(r)}=o(1) \quad(r \rightarrow \infty) .
\end{array}
$$

To complete the proof, we estimate the contribution from pairs of intervals $\mathbf{J} \in \mathcal{I}_{3}^{\prime}$; each of those intervals have nonempty intersection with $\mathcal{B}$. We recall (9) once again and divide this annulus into congruent regions (wedges) obtained by intersecting $\mathcal{B}$ with sectors of angular opening $O\left(L^{-1 / 3}(r)\right.$ ), oriented so that $z$ itself lies on the bisector of one of these regions (wedges). As before, the number of intervals of $\Gamma^{\sharp}$ in each sector is $O(L(r))$. Let $\Omega(z)$ be the wedge which contains $z$.

If $\left(J \cup J^{*}\right) \cap \Omega(z)=\varnothing$, so $z$ is separated from $J$ and $J^{*}$ by $1 \leq \ell \leq O\left(L^{1 / 3}(r)\right)$ sectors, then

$$
\frac{|J|}{\left|z-\zeta_{J}\right| \wedge\left|z-\zeta_{J^{*}}\right|} \leq C \frac{r / L(r)}{\ell r L^{-1 / 3}(r)}=\frac{C}{\ell L^{2 / 3}(r)}
$$

and each sector contains $O(L(r))$ intervals of $\Gamma^{\sharp}$. For simplicity write $\mathbf{J} \subset \Omega(z)$ if $\mathbf{J}=\left(J, J^{*}\right)$ and either $J$ or $J^{*}$ intersects $\Omega(z)$. Then summing for $\mathbf{J} \subset \mathcal{I}_{3}^{\prime} \backslash \Omega(z)$, we have

$$
\sum_{\mathbf{J} \subset \mathcal{I}_{3}^{\prime} \backslash \Omega(z)}\left(\frac{|J|}{\left|z-\zeta_{J}\right| \wedge\left|z-\zeta_{J^{*}}\right|}\right)^{2} \leq C \frac{L(r)}{L^{4 / 3}(r)} \sum_{1}^{\infty} \frac{1}{\ell^{2}}=o(1) \quad(z \rightarrow \infty) .
$$

Next, consider the sum over pairs of intervals such that one member of the pair intersects $\Omega(z)$. Divide $\Omega(z)$ into disjoint subregions $\Omega_{\ell}(z)$ using circles centered at $z$ of radius $\ell r / L(r), \ell \in \mathbb{N}$. Now since $\mu(J)=1\left(\right.$ or $\mu^{*}\left(J^{*}\right)=1$ ) then $|J|=\left|J^{*}\right|=$ 
$\operatorname{cr} / L(r)$ and therefore the number of intervals in each $\Omega_{\ell}(z)$ is uniformly bounded. Since (39) holds, we have $\ell \geq 2$, and so

$$
\sum_{\ell \geq 2} \sum_{\mathbf{J} \subset \Omega_{\ell}(z)}\left(\frac{|J|}{\left|z-\zeta_{J}\right| \wedge\left|z-\zeta_{J^{*}}\right|}\right)^{2} \leq C \sum_{\ell \geq 2}\left(\frac{r / L(r)}{\ell r / L(r)}\right)^{2}=C \sum_{\ell \geq 2} \frac{1}{\ell^{2}}<\infty .
$$

where again we write $\mathbf{J} \subset \Omega_{\ell}(z)$ if $\mathbf{J}=\left(J, J^{*}\right)$ and $\left(J \cup J^{*}\right) \cap \Omega_{\ell}(z) \neq \varnothing$.

That the estimate of Lemma 4 is not $o(1)$ is due to the term (40), but if (39) is replaced by the stronger (44) we get the more flexible (43), which is the key to $\S 4$.

Corollary 1. For fixed $K \geq 15$ and fixed $z_{0}$, with $\left|z_{0}\right|$ so large that

$$
2 K<L^{1 / 3}\left(\left|z_{0}\right|\right)
$$

let

$$
\begin{aligned}
& \mathrm{D}\left(z_{0}\right)=\left\{\zeta:\left|\zeta-z_{0}\right|<5\left|z_{0}\right| / L\left(\left|z_{0}\right|\right)\right\} \\
& \mathrm{D}^{\prime}\left(z_{0}\right)=\left\{\zeta:\left|\zeta-z_{0}\right|<10 K\left|z_{0}\right| / L\left(\left|z_{0}\right|\right)\right\}
\end{aligned}
$$

and let

$$
\mathcal{I}^{\star}=\left\{\mathbf{J}=\left(J, J^{*}\right): \mathrm{D}^{\prime}\left(z_{0}\right) \cap\left(J \cup J^{*}\right) \neq \varnothing\right\} .
$$

Then, with $h_{\mathbf{J}}$ from (38),

$$
|\log | g(z)\left|-U(z)-\sum_{\mathbf{J} \in \mathcal{I}^{\star}} h_{\mathbf{J}}(z)\right| \leq C K^{-1}+o(1), \quad z \in \mathrm{D}\left(z_{0}\right) .
$$

Proof. The only term in Lemma 4 not $o(1)$ is (40), so by increasing the radius of the ball in (39) one gets a better estimate. Concretely, if $\left|z_{0}\right|$ is large enough and $\left(J, J^{*}\right) \notin \mathcal{I}^{\star}$ then $d\left(z_{0}, \zeta_{J} \cup \zeta_{J^{*}}\right) \geq 5 K\left|\zeta_{J}\right| / L\left(\left|\zeta_{J}\right|\right)$ and therefore

$$
d\left(z, \zeta_{J} \cup \zeta_{J^{*}}\right) \geq \frac{5 K\left|\zeta_{J}\right|}{2 L\left(\left|\zeta_{J}\right|\right)} \quad\left(z \in \mathrm{D}\left(z_{0}\right)\right)
$$

For $z \in \mathrm{D}\left(z_{0}\right)$, follow the proof of Lemma 4 but now summing over intervals that are not in $\mathcal{I}^{\star}$. According to (44), $\ell>K$ in (40) so the sum over the $\mathbf{J} \subset \Omega(z)$, where $\Omega(z)$ is the wedge introduced in the lemma, is bounded by $C \sum_{\ell>K} \ell^{-2}=O\left(K^{-1}\right)$ while the sums over all the intervals not in $\Omega(z)$ remain the same. Therefore

$$
\sum_{\mathbf{J} \notin \mathcal{I}^{\star}}\left|h_{\mathbf{J}}(z)\right| \leq C K^{-1}+o(1), \quad z \in \mathrm{D}\left(z_{0}\right) .
$$

Since

$$
|\log | g(z)\left|-U(z)-\sum_{\mathbf{J} \in \mathcal{I}^{\star}} h_{\mathbf{J}}(z)\right| \leq \sum_{\mathbf{J} \notin \mathcal{I}^{\star}}\left|h_{\mathbf{J}}(z)\right|+\left|V_{e}(z)\right|,
$$

lemma 2 and the estimation above give (43).

3.5. Estimates near the exceptional set $E$ : Proof of Theorem $\mathbf{2}^{\prime}(c)$. Lemma 5 below complements Lemma 3 when (39) fails. For now we still assume that the component of $E \ni z$ is a single disk, as in hypothesis $(c)$. Together, the two lemmas of this section imply assertion $(c)$ of Theorem $2^{\prime}$.

Lemma 5. Let $z \in \Omega:=B\left(\zeta_{J}, 3\left|\zeta_{J}\right| / L\left(\left|\zeta_{J}\right|\right)\right)$ where $J$ is an interval of $\Gamma^{0} \subset \Gamma^{\sharp}$ of $\mu$-measure one. Let $J^{*}=-J$ and $\mathbf{J}=\left(J, J^{*}\right)$. Then, with $h_{\mathbf{J}}$ from (38), $h_{\mathbf{J}}(z)<C$, $C$ an absolute constant.

Equivalently, if $z \in B\left(\zeta_{J^{*}}, 3\left|\zeta_{J^{*}}\right| / L\left(\left|\zeta_{J^{*}}\right|\right)\right.$, with $J^{*} \in \Gamma^{*} \subset \Gamma^{\sharp}$ let $J=-J^{*}$ and $\mathbf{J}=\left(J, J^{*}\right)$. Then $h_{\mathbf{J}}(z)>-C$. 
Note that the disk $\Omega=\Omega\left(\zeta_{J}\right)$ is somewhat larger than those in $E$ (31); the disks $\Omega\left(\zeta_{J}\right)$ are no longer disjoint.

Proof. We consider only the first assertion, and note that there can only be an upper bound, since $h_{\mathbf{J}}\left(\zeta_{J}\right)=-\infty$.

Let $|z|=r$. It is elementary, from (34) and the fact that $z, \zeta \in \Omega$, that

$$
\begin{aligned}
h_{\mathbf{J}}(z) & =\log \left|z-\zeta_{J}\right|-\int_{J} \log |z-\zeta| d \mu(\zeta)+o(1) \\
& \leq \log \frac{r}{L(r)}-\int_{J} \log |z-\zeta| d \mu(\zeta)+O(1) \quad(z \in \Omega),
\end{aligned}
$$

and since $\int_{J} \log |z-\zeta| d \mu(\zeta)$ is harmonic in $\Omega \backslash J$, Lemma 3 applies for $z \in \partial \Omega$. By the maximum principle, we need only bound the integral when $z \in J$.

We suppose that $J \subset \mathbb{R}^{+}$, and let $t \in J$. Set $I=J r^{-1}$ (where $|z|=r$ ) and choose $s \in I$ with $s=t r^{-1}$. According to (17), $d \mu=2 r^{-1} L(r) d r$ on $J$, and so

$$
\int_{J} \log |z-\zeta| d \mu(\zeta)=\log r+2 \int_{I} L(r s) \log |s-1| s^{-1} d s+o(1) .
$$

By (7) and (33), $L(r s)=L(r)+o(1) \log (s / r)=L(r)+o(1) L(r)^{-1}$, the $o(1)$ uniform in $s \in I$. If $I=\left[1-c_{1} / L(r), 1+c_{2} / L(r)\right]$, the condition $\mu(J)=1$ implies that $c_{1}+c_{2}=1 / 2+O\left(L^{-1}(r)\right)$. Since $u \log u$ decreases for $u<e^{-1}$, we have

$$
\begin{aligned}
0 \geq \int_{I} L(r s) \log |s-1| & s^{-1} d s=\left(L(r)+o(1) L^{-1}(r)\right) \int_{I} \log |s-1| s^{-1} d s \\
& =\left(L(r)+o(1) L^{-1}(r)\right)\left(1+O\left(L^{-1}(r)\right)\right) \int_{I} \log |s-1| d s \\
& =(L(r)+O(1)) \int_{I} \log |s-1| d s \\
& =(L(r)+O(1)) \frac{1}{2}\left(L^{-1}(r) \log \left(L^{-1}(r)\right)+O\left(L^{-1}(r)\right)\right) \\
& =\left(c_{1}+c_{2}\right) \log L^{-1}(r)+O(1)=-\frac{1}{2} \log L(r)+O(1),
\end{aligned}
$$

which we then insert in (46) and then (45).

Finally we consider the situation that $z \in \Omega$, but not too near $\zeta_{J}$.

Lemma 6. For $\lambda>0$, let $z \in \Omega$ as in Lemma 5 with

$$
\lambda \frac{\left|\zeta_{J}\right|}{L\left(\left|\zeta_{J}\right|\right)} \leq\left|z-\zeta_{J}\right| \wedge\left|z-\zeta_{J^{*}}\right| \leq 3 \frac{\left|\zeta_{J}\right|}{L\left(\left|\zeta_{J}\right|\right)}
$$

Then $\left|h_{\mathbf{J}}(z)\right|<C=C(\lambda)$.

Proof. Let $|z|=r$ and note that (47) shows that $z \in B\left(\zeta_{J}, 10 r / L(r)\right)$. Thus

$$
\log \frac{r}{L(r)}-C(\lambda) \leq \log \left|z-\zeta_{J}\right| \leq \log \frac{r}{L(r)}+C,
$$

so the proof of Lemma 5 shows the expression in the first line of (45) is uniformly bounded. 
3.6. Statement and proof of Theorem 3: controlling behavior on E. We exploit the special forms of $U$ and $\Gamma^{0}$ near the inner boundaries of each $\mathcal{A}_{k}$, as described in $\S 2.5$, to give bounds for $V_{\Gamma}^{\sharp}$ on $E$ for the situations not settled in Theorem 2. The proofs rely on techniques used in Theorem 2 (Theorem $2^{\prime}$ ).

Theorem 3. Let the assumptions and notations of Theorem 2 remain in force, augmented by (25)-(28). Then we also have

(a) the components $E^{\prime}$ of $E$ are either single disks or the union of three disks. In the latter case, $E^{\prime}$ contains three point masses, one of which is a zero and one a pole of $g$,

(b) if $z \in E^{\prime}$ where $E^{\prime}$ is a component of $E$ containing centroids $\zeta_{I}, \zeta_{J}, \zeta_{K}$, atoms of the approximating measure $\sigma-\sigma^{*}$, with $\zeta_{I}$ a zero of $g$ and $\zeta_{J}$ a pole, then with $C_{0}$ the constant of Theorems 2 or $\mathscr{2}^{\prime}$

$$
\begin{aligned}
V_{\Gamma^{\sharp}}(z) \leq C_{0} & \text { if }\left|\zeta_{I}-z\right| \leq\left|\zeta_{J}-z\right| \text { and } \zeta_{K} \text { is a zero of } g, \\
V_{\Gamma^{\sharp}}(z) \geq-C_{0} & \text { if }\left|\zeta_{I}-z\right| \leq\left|\zeta_{J}-z\right| \text { and } \zeta_{K} \text { is a pole of } g .
\end{aligned}
$$

Proof. Since (28) holds when $z \in \mathcal{K}\left(\mathcal{K}\right.$ from (24)), if a component of $E^{\prime}$ consists of more than one disc, it must intersect $\left\{r_{k} \leq|z| \leq \rho_{k}\right\}$ for some (large) $k$. For convenience, let us assume that $E^{\prime} \subset \mathcal{U}$. According to (17), the centers $\left\{\zeta_{p}\right\}$ of all disks contained in $E^{\prime}$ have the same modulus, and since (34) holds, (31) shows that disks corresponding to point measures which intersect a single arc $\gamma \cap \mathcal{A}_{k}, \gamma \subset \Gamma^{*}$, are disjoint. By (27), three branches of $\Gamma^{\sharp} \cap \mathcal{A}_{k}^{o}$ emerge from each bifurcation node $\pm z_{k} \in S\left(r_{k}\right)$ (since $E^{\prime} \subset \mathcal{U}$, there are two in $\Gamma^{0}$ and one in $\Gamma^{*}$ ) and separate uniformly as $r$ increases. Hence components $E^{\prime}$ associated to these branches consist of one ball or three balls, in the latter case two associated to a zero of $g$, and the other to a pole. This proves claim $(a)$.

In considering $(b)$. Let $E^{\prime}$ be the component of $E$ containing centroids $\zeta_{I}, \zeta_{J}, \zeta_{K}$, where $\zeta_{I}$ is a zero of $g$ (i.e. $I \in \Gamma^{0}$ where $\zeta_{I}$ centroid of $I$ ) and $\zeta_{J}$ a pole (i.e. $J \in \Gamma^{*}$ ). Let $K$ be the interval in $\Gamma^{\sharp}$ with centroid $\zeta_{K}$, and finally consider the sets of pair of intervals $\mathbf{I}, \mathbf{J}$ and $\mathbf{K}$ formed by the intervals $I, J, K$ and their negative counterparts $-I,-J,-K$ ordered as in Lemma 3 . When $E^{\prime} \subset \mathcal{U}, g\left(\zeta_{K}\right)=0$. Using the notation in (38) we show for some absolute constant $C$ that if $z \in E^{\prime}$ then

$$
h_{\mathbf{I}}(z)+h_{\mathbf{J}}(z)+h_{\mathbf{K}}(z) \leq C, \quad\left(z \in E^{\prime},\left|\zeta_{I}-z\right| \leq\left|\zeta_{J}-z\right|\right)
$$

with the opposite estimate when $g\left(\zeta_{K}\right)=\infty$. Once (48) is proved, the estimate in (b) follows from Lemma 4 together with (48) applied to the terms which fail to satisfy (39), as we did at the beginning of $\S 3.5$ in Lemma 5 .

Let $r=|z|, \gamma$ be the arc of $\Gamma^{0}$ associated to $\zeta_{K}$, and let $\zeta \in \gamma,|\zeta|=t$. Then $S(t)$ meets arcs $\gamma^{\prime} \subset \Gamma^{0} \cap E$ (associated to $\zeta_{I}$ ) and $\gamma^{*} \subset \Gamma^{*} \cap E$ (corresponding to $\left.\zeta_{J}\right)$ at $\zeta^{\prime}, \zeta^{*}$, and $\gamma, \gamma^{\prime}$ and $\gamma^{*}$ meet at a bifurcation node $z_{k}$ of $\Gamma^{\sharp}$. Since we have assumed that $\left|\zeta_{I}-z\right| \leq\left|\zeta_{J}-z\right|$, the strict condition (27) near the bifurcation node $z_{k}$ ensures that

$$
|\log | \frac{z-\zeta_{J}}{z-\zeta_{K}}||=O(1), \quad|\log | \frac{z-\zeta^{*}}{z-\zeta}||=O(1) .
$$

Hence $\left|\zeta_{I}-z\right|<\left|\zeta_{J}-z\right|\left(<\left|\zeta_{K}-z\right| \mid\right)$ and so $h_{\mathbf{I}}(z)+h_{\mathbf{J}}(z)+h_{\mathbf{K}}(z)=h_{\mathbf{I}}(z)+O(1)$. The result now follows from Lemma 5 . 


\section{ON THE IMAGINARY PARTS}

4.1. Two key cases. To identify the possible asymptotic curves of $g$, it is clear that more is needed than data on $|g|$. We prove

Theorem 4. The only possible asymptotic values of $w=g(z)$ are 0 and $\infty$. Moreover, if $\eta$ is any asymptotic path for $w=0$, then there is a curve $\gamma \subset \Gamma^{0} \subset \Gamma^{\sharp}$ on which $g \rightarrow 0$, such that for each $\varepsilon>0$, the set $\{|g(z)|<\varepsilon\}$ contains a component $\Omega$ so that $\eta$ and $\gamma$ are in $\Omega \cap\left\{|z|>r^{\prime}\right\}$ if $r^{\prime}$ is sufficiently large. Thus $\eta$ and $\gamma$ belong to the same tract corresponding to $w=0$.

A similar statement holds with $w=0$ replaced by $w=\infty$.

Thus consider a (hypothetical) curve $\eta$ tending to $z=\infty$ on which $g(z) \rightarrow a$, so that $|g|$ is nearly constant on $\eta$ (if $a \neq \infty$ ). Using the notation from (42), we consider a family of disks $\mathrm{D}^{\prime}\left(z_{0}\right)$, with $z_{0} \in \mathcal{K} \cap \eta$ (recall (24)) through which $\eta$ would have to pass. Let us denote by $\mathcal{D}_{\eta}$ such a family. Comment. The points $z_{0}$ should not be confused with the first node $z_{0}$ of the network $\Gamma^{0}$.)

Let $K$ be fixed (and large) with $z_{0} \in S\left(r_{0}\right) \cap \mathcal{K},\left|z_{0}\right|=r_{0}$ so large that (41) holds $\left(r_{0}\right.$ should not be confused with the inner boundary of $\mathcal{A}_{0}$ from (6)). Since $z_{0} \in \mathcal{K}$, (28) implies that $\mathrm{D}^{\prime}\left(z_{0}\right)$ intersects at most one curve from $\Gamma^{\sharp}$. Thus $\mathrm{D}^{\prime}\left(z_{0}\right)$ meets at most two regions $\Delta$ in $\mathbb{C} \backslash \Gamma^{\sharp}$, and so for each disk $\mathrm{D}^{\prime}\left(z_{0}\right)$ there are two posibilities:

(a) $\mathrm{D}\left(z_{0}\right) \cap \Gamma^{\sharp}=\varnothing$ for $\mathrm{D}\left(z_{0}\right) \subset \mathrm{D}^{\prime}\left(z_{0}\right)$ (see $\left.(42)\right)$ or,

(b) $\mathrm{D}\left(z_{0}\right)$ contains an $\operatorname{arc} \gamma \subset \Gamma^{\sharp}$,

and then two situations could occur:

(i) There are infinitely many disks in $\mathcal{D}_{\eta}$ for which posibility $(a)$ holds,

(ii) there are only a finite number of disks in $\mathcal{D}_{\eta}$ for which $(a)$ holds.

When $\eta$ is far from $\Gamma^{\sharp}$ (case $\left.(i)\right)$ and $z \in \eta$, we may suppose that $\log |g(z)|$ is close to the model function (cf. (20)) on $S(|z|) \cap \mathrm{D}\left(z_{0}\right)$. When $\eta$ is near $\Gamma^{\sharp}$ is far more delicate; details are in $\S 4.3$. Since the curves of $\Gamma^{\sharp}$ are asymptotically rays when $z \in \mathcal{K}$, we assume that $\gamma$ is the positive real axis.

4.2. Proof of Theorem 4 (start). Let $g \rightarrow a$ on a curve $\eta$. If $a=0, \infty$, we will associate a curve $\gamma \subset \Gamma^{*}$ 'near' $\eta$ on which also $g \rightarrow a$. To eliminate the possibility $a \neq 0, \infty$ is harder, and for that we need the rest of this section (for case $(i)$ ) and the next (case $(i i))$.

Now let $\eta$ be an asymptotic curve of $g$, so that $g(z) \rightarrow a$ as $z \rightarrow \infty$ on $\eta$.

First suppose $a=0$ or $\infty$; say $a=0$. In case $(i)$, choose $r_{0}$ large and $z_{0} \in$ $\eta \cap S\left(r_{0}\right)$, so that $(a)$ holds for $\mathrm{D}^{\prime}\left(z_{0}\right)$. We may assume using Theorem 2 or $2^{\prime}$ that if $S(r) \cap \mathrm{D}\left(z_{0}\right) \neq \varnothing$, then in the component $\Omega\left(z_{0}\right)$ of $\mathbb{C} \backslash \Gamma^{\sharp}$ which contains $z_{0}, \log |g|$ is close to a model function $U$ of (20), and thus is linear in $\arg z$. Hence we obtain an arc of $S\left(r_{0}\right)$ joining $z \in S\left(r_{0}\right) \cap \eta$ to $\Gamma^{\sharp}$ with $U\left(r_{0} e^{i \theta}\right)$ having its maximum at $z$ and decreasing on this arc until reaching a minimum at $\Gamma^{\sharp}$ (outside $\mathrm{D}\left(z_{0}\right)$ ). It follows that any component of $\{U<-M\}$ which meets $\eta$ on $S\left(r_{0}\right)$ for large $r_{0}$ also intersects some curve $\gamma \subset \Gamma^{0}$, and so if $g \rightarrow 0$ on $\eta$, then $g \rightarrow 0$ on $\gamma$. Analogous comments apply when $a=\infty$.

In case $(i i)$ an even easier argument works, since $\eta$ is already close to a single $\operatorname{arc}$ of $\Gamma^{*}$.

More subtle is that $0, \infty$ are the only possible asymptotic values. Let $\eta$ be a curve on which $g \rightarrow a \neq 0, \infty$. 
If case $(i)$ applies, let $\mathrm{D}^{\prime}\left(z_{0}\right)$ be a disk for which $(a)$ holds, then $\log |g|$ and $\theta=\arg z$ are harmonic in $\mathrm{D}^{\prime}\left(z_{0}\right)$. We suppose that near $z_{0}, U$ is given by $(20)$. Thus given $\epsilon>0$, if $K$ and $\left|z_{0}\right|$ are large $\left(z_{0} \in \eta \cap \mathcal{K}\right)$ then by (43), (7) and (15),

$$
\begin{aligned}
& \left|\left(\log |g(z)|-\left(A+\tau L\left(r_{0}\right) \theta\right)\right)\right|:=\left|\epsilon^{\prime}(z)\right|<\epsilon \quad\left(z \in \mathrm{D}^{\prime \prime}\left(z_{0}\right)\right), \\
& \left|\arg g(z)-\left(A^{\prime}-\tau L\left(r_{0}\right) \log r\right)\right|:=|\epsilon(z)|<\epsilon \quad\left(z \in \mathrm{D}^{\prime \prime}\left(z_{0}\right)\right)
\end{aligned}
$$

for suitable constants $A, A^{\prime}, \tau \in\{ \pm 1\}$ and $\mathrm{D}^{\prime \prime}\left(z_{0}\right)$ the disk centered at $z_{0}$ with radius half of that of $\mathrm{D}\left(z_{0}\right)$ (in fact, the first line holds in the larger $\mathrm{D}\left(z_{0}\right)$ ). The second line (which restates the first for the conjugate functions) holds in $\mathrm{D}^{\prime \prime}\left(z_{0}\right)$ since $K$ is large. By hypothesis, $\log |g|=\log |a|+o(1)$ on $\eta$ and $z_{0}=r_{0} e^{i \theta_{0}} \in \eta$. Thus (20) and the first line of (49) show that $\left|\theta-\theta_{0}\right|=O\left(\epsilon L^{-1}\left(r_{0}\right)\right)$ in $\eta \cap \mathrm{D}^{\prime \prime}\left(z_{0}\right)$. However, on $\left\{\arg z=\theta_{0}\right\} \cap \mathrm{D}^{\prime \prime}\left(z_{0}\right)$, the function $\log r$ increases by more than $2 / L\left(r_{0}\right)$. The second estimate of (49) with $\epsilon$ small and $K$ large but fixed then implies that $\arg g(z)$ varies by at least $\pi / 2$ on $\eta \cap \mathrm{D}^{\prime \prime}\left(z_{0}\right)$. In other words, if $a \neq 0, \infty, \eta$ will contain points in $\mathrm{D}^{\prime \prime}\left(z_{0}\right)$ whose $g$-images are well-separated on $\{|w|=a\}$, and so $g$ cannot be uniformly close to $a$ on $\eta \cap \mathrm{D}^{\prime \prime}\left(z_{0}\right)$.

4.3. Case (ii). This situation is more difficult. Again $g(z) \rightarrow a \neq 0, \infty$ on $\eta$, but we assume that whenever $z_{0} \in \eta \cap \mathcal{K}$ with $\left|z_{0}\right|$ sufficiently large, $\mathrm{D}\left(z_{0}\right) \cap \Gamma^{\sharp} \neq \varnothing$. By (28) and (41) $\mathrm{D}\left(z_{0}\right) \cap \Gamma^{\sharp}$ consists of portions of one arc $\gamma$; for specificity, take $\gamma \subset \Gamma^{0}$. Due to (29), $\gamma \cap \mathrm{D}^{\prime}\left(z_{0}\right)$ is a ray which contains the centroids $\zeta_{I} \in \mathrm{D}^{\prime}\left(z_{0}\right)$

In contrast to case $(i)$, the geometry of $\eta$ is not apparent. An insightful example is $w=\sin z$, where $\Gamma^{\sharp}=\mathbb{R}$. The level-set $\{|\sin z|=1\}=\{\pi / 2 \pm k \pi, k \in \mathbb{Z}\}$, is a 'necklace' of topological circles meeting tangentially at the critical points. Thus, by moving alternately in the upper and lower half-planes, we find a curve $\eta$ which $|\sin z|=1$, but $\arg (\sin z)$ never varies more than $\pi$.

Write $\gamma \cap \mathrm{D}^{\prime}\left(z_{0}\right)=\cup_{\mathcal{I}} I$, where each $I$ has mass one (this may require slightly modifying $\left.\partial \mathrm{D}^{\prime}\left(z_{0}\right)\right)$. Since $d \mu=2 r^{-1} L(r)$ on $\gamma$, there are at most $O(K)$ centroids $\zeta_{I}$ with $I \in \mathcal{I}$.

It is also useful to extend $\gamma$ in both directions to separate $\mathbb{C}$ (as well as $\mathrm{D}^{\prime}\left(z_{0}\right)$ and $\mathrm{D}\left(z_{0}\right)$ ) into two components: $\gamma$ an interval on $\mathbb{R}$.

Let $\mathrm{D}^{\prime+}\left(z_{0}\right), \mathrm{D}^{\prime-}\left(z_{0}\right)$ be the two components of $\mathrm{D}^{\prime}\left(z_{0}\right) \backslash \gamma$, and in each of $\mathrm{D}^{\prime \pm}\left(z_{0}\right)$ take branches of $\arg (z-\zeta)(\zeta \in \gamma), \arg \left(z-\zeta_{I}\right)(I \in \mathcal{I})$. Similarly let $\mathrm{D}^{ \pm}\left(z_{0}\right) \subset$ $\mathrm{D}^{\prime \pm}\left(z_{0}\right)$ be the components of $\mathrm{D}\left(z_{0}\right) \backslash \gamma$. Using notation from Corollary 1 (§3.4), consider $\mathcal{I}^{\star}=\{\mathbf{I}=(I,-I): I \in \mathcal{I}\}$ and write $\mathcal{H}(z)=\sum_{\mathcal{I}^{\star}} h_{\mathbf{I}}(z)$ for $z \in \mathrm{D}\left(z_{0}\right)$ and $h_{\mathbf{I}}$ from (38). One utility of assumption (29) is that we have explicit expressions for $\mathcal{H}$ and its conjugate $\tilde{\mathcal{H}}$, the latter defined in each component of $\mathrm{D}^{\prime}\left(z_{0}\right)$ :

$$
\begin{aligned}
& \mathcal{H}(z)=\sum_{\mathcal{I}^{\star}} h_{\mathbf{I}}(z)=\sum_{\mathcal{I}} \log \left|1-z / \zeta_{I}\right|-2 \int_{\gamma \cap \mathrm{D}^{\prime}\left(z_{0}\right)} \log |1-z / t| t^{-1} L(t) d t+o(1), \\
& \tilde{\mathcal{H}}(z)=\sum_{\mathcal{I}^{\star}} \tilde{h}_{\mathbf{I}}(z)=\sum_{\mathcal{I}} \arg \left(1-z / \zeta_{I}\right)-2 \int_{\gamma \cap \mathrm{D}^{\prime}\left(z_{0}\right)} \arg (1-z / t) t^{-1} L(t) d t+o(1),
\end{aligned}
$$

where $o(1)$ accounts for the contribution from $-\left(\gamma \cap \mathrm{D}^{\prime}\left(z_{0}\right)\right)$, which lies far from $z_{0}$.

Lemma 7. Let $\tilde{\mathcal{H}}^{ \pm}$be a suitable branch in each component of $\mathrm{D}^{\prime}\left(z_{0}\right) \backslash \gamma$, and let $p, q \in \gamma \cap \partial \mathrm{D}^{\prime}\left(z_{0}\right)$. Then

$$
\begin{aligned}
& \left\|\tilde{\mathcal{H}}^{ \pm}\right\|_{\infty} \leq \pi / 2 \\
& \tilde{\mathcal{H}}^{+}(q+i 0)-\tilde{\mathcal{H}}^{+}(p+i 0)=-\left[\tilde{\mathcal{H}}^{-}(q-i 0)-\tilde{\mathcal{H}}^{-}(p-i 0)\right] .
\end{aligned}
$$


Proof. This is straightforward. Each function $\tilde{\mathcal{H}}^{ \pm}$is a sum of a finite number of terms $h_{\mathbf{I}}$ from (38). If $\zeta \in \gamma$ (possibly $\zeta=\zeta_{I}, I \in \mathcal{I}$ ), then $\arg (z-\zeta)=0$ when $z \in \gamma, z>\zeta$ (using language inherited from viewing $\gamma \subset\{\Re z>0\}$ ), while $\arg (z-\zeta)= \pm \pi$ when $z \in \gamma, z<\zeta$; the sign depending on the function $\tilde{\mathcal{H}}^{ \pm}$ under scrutiny. Thus, the boundary values of the conjugate $\tilde{h}_{I}$ of any single term $\log \left|z-\zeta_{I}\right|-\int_{I} \log |z-\zeta| d \mu(\zeta)$ are zero for $z \in \gamma \backslash\{I\}$. This remark also justifies the other assertion.

To adapt (49) to the situation (ii), let $K, z_{0}$ be large, $z_{0} \in \eta$, subject to (41). The left side of $(43)$ is harmonic in $\mathrm{D}^{\prime}\left(z_{0}\right)$, since $\mathcal{H}$ cancels the Riesz mass. Hence we make take conjugates, with constant $A$ in $\mathrm{D}^{\prime}\left(z_{0}\right)$ and constants $A^{\prime}$ in $\mathrm{D}^{\prime \pm}\left(z_{0}\right)$ :

$$
\left\{\begin{array}{l}
\left|\left(\log |g|-\left(A+\tau L\left(r_{0}\right)|\theta|\right)+\mathcal{H}(z)\right)\right|:=\left|\epsilon^{\prime}(z)\right|<\epsilon \quad\left(z \in \mathrm{D}\left(z_{0}\right)\right), \\
\arg g(z)-\left(A^{\prime} \mp \tau L\left(r_{0}\right) \log r+\tilde{\mathcal{H}}^{ \pm}(z)\right)|:=| \epsilon(z) \mid<\epsilon \quad\left(z \in \mathrm{D}^{ \pm}\left(z_{0}\right)\right) .
\end{array}\right.
$$

Lemma 8. Let $\eta$ be a curve on which $g(z) \rightarrow$ a such that $\eta$ passes through the center $z_{0}$ of $\mathrm{D}\left(z_{0}\right)$ with $\Gamma^{\sharp} \cap \mathrm{D}\left(z_{0}\right) \neq \varnothing$. Then $\eta$ contains an arc $\eta^{\prime}$ on which $\arg g(z)$ varies by at least $\pi / 2$. Hence if $a \neq 0, \infty, g$ cannot be uniformly close to a on all of $\eta$.

Proof. Recall that we are in case $(i i)$. We consider two possibilities.

First, suppose there is a subarc $\eta_{1} \subset \eta$, with $\eta_{1} \cap \gamma=\varnothing$ which is not insignificant, in the sense that its extremes are points $\zeta_{1}, \zeta_{2}$ in $\mathrm{D}\left(z_{0}\right)$ with $\log \left(\left|\zeta_{2} / \zeta_{1}\right|\right)>$ $4 \pi\left(L\left(r_{0}\right)\right)^{-1}$. We then consider the second estimate of (51) at each $\zeta \in \eta^{\prime}$ relative to $\mathrm{D}^{ \pm}\left(z_{0}\right)$ as appropriate, using some branch of $\arg g\left(\zeta_{1}\right)$. We reach a contradiction since $L\left(r_{0}\right) \log r$ has changed by at least $3 \pi$ while (by $\left.(50)\right)\|\tilde{\mathcal{H}}\|_{\infty} \leq \pi / 2$. Once again, $\arg g(z)$ cannot be nearly constant on $\eta^{\prime}$.

The more subtle case is when there is no significant subarc of $\eta$ in any $\mathrm{D}^{\prime}\left(z_{0}\right) \backslash \gamma$ (as with $w=\sin z$ ). Let

$$
P(\eta)=\eta \bigcap\left(\gamma \cap \mathrm{D}\left(z_{0}\right)\right)
$$

With $s>0$ small but fixed, we have that $P(\eta) \cap\left(\cup_{\mathcal{I}} B\left(\zeta_{I}, s\right)\right)=\varnothing$, and may assume that $P(\eta)$ is discrete in $\gamma$. Suppose $\eta$ contains a subarc $\eta^{\prime}$ having only its endpoints $\xi, \xi^{\prime}\left(\left|\xi^{\prime}\right|>|\xi|\right)$ in $P(\eta)$, such that the (closure of the) domain (in one of $\mathrm{D}^{+}\left(z_{0}\right)$ or $\left.\mathrm{D}^{-}\left(z_{0}\right)\right)$ bounded by $\eta^{\prime}$ and a subarc $\hat{\gamma} \subset \gamma$ contains at least one $\zeta_{I}$, say $\left\{\zeta_{I}: I \in \mathcal{I}^{\prime}\right\}$.

We claim that $\eta^{\prime}$ contains a subarc on which $\arg g(\zeta)$ varies by more than a fixed amount. Thus, we compute $\arg g\left(\xi^{\prime}\right)-\arg g(\xi)$ in the second formula of (51) in each of $\mathrm{D}^{ \pm}\left(z_{0}\right) \backslash \gamma$, since one of these computations is with the change of $\arg g$ on $\eta^{\prime}$.

Lemma 7 shows that the change, on $\left[\xi, \xi^{\prime}\right]$ relative to $\mathrm{D}^{+}\left(z_{0}\right)$, of the sum

$$
-L\left(r_{0}\right) \log r+\tilde{\mathcal{H}}^{+}
$$

in $\mathrm{D}^{+}\left(z_{0}\right)$ is the negative of that of the sum $+L\left(r_{0}\right) \log r+\tilde{\mathcal{H}}^{-}$in $\mathrm{D}^{-}\left(z_{0}\right)$. But the second line of (51) shows that each of these is (up to $o(1)$ ) the change of $\arg g(z)$.

Finally, a closed curve consisting of simple arcs from $\xi$ to $\xi^{\prime}$ in $\mathrm{D}^{+}\left(z_{0}\right)$ and then $\mathrm{D}^{-}\left(z_{0}\right)$ form a closed curve on which the change of $\arg g$ is $2 \pi \operatorname{card}\left(\mathcal{I}^{\prime}\right)$. That means that $\left|\arg g\left(\xi^{\prime}\right)-\arg g(\xi)\right|$, when computed relative to $\mathrm{D}^{ \pm}\left(z_{0}\right)$, is well-defined up to $o(1)$, and is at least $\pi$ card $\left(\mathcal{I}^{\prime}\right)$. Thus $\arg g(z)$ cannot be nearly constant on all of $\eta^{\prime}$ if $a \neq 0, \infty$.

This completes the proof of Theorem 4 . 
4.4. The asymptotic values. It is easy to guarantee that $\operatorname{As}(g)=\{0, \infty\}$.

Lemma 9. Suppose there is a curve $\gamma \subset \Gamma^{0} \cap \mathcal{U}$, on which $U(z) \rightarrow-\infty$. Then $A s(g)=\{0, \infty\}$.

Proof. By Theorem $2^{\prime}, \log |g(z)| \leq U(z)+C_{0}$ if $z \in \gamma \backslash E$ or if $z \in \gamma \cap E$ and the component of $E$ containing $z$ consists of a single ball centered at a zero of $g$. So by the construction in $\S 2.5$ we only need consider the situation that the component $E^{\prime}$ of $E$ containing $z$ consists of three balls: $E^{\prime}$ contains two zeros and one pole of $g$. Let $z_{c} \in E^{\prime}$ be the zero of $g$ associated to $\gamma$ and $z_{p} \in E^{\prime}$ a pole. Elementary geometry shows that $\left|z_{c}-z\right| \leq\left|z_{p}-z\right|$ when $z \in \gamma$. Thus by Theorem $2(e)$,

$$
\log |g(z)| \leq U(z)+C_{0}, \quad(z \in \gamma)
$$

and since $U \rightarrow-\infty$ on $\gamma$,

$$
\log |g(z)| \rightarrow-\infty, \quad(z \rightarrow \infty, z \in \gamma)
$$

Now with $\gamma$ as above, let $\gamma^{\prime}=-\gamma$ be a second curve on $\Gamma^{*} \subset \Gamma^{\sharp}$. Since $U(z)=$ $-U(-z)((4)), U \rightarrow \infty$ on $\gamma^{\prime}$, and our argument shows that $\log |g| \rightarrow \infty$ on $\gamma^{\prime}$.

Let $\Gamma$ be the subnetwork of $\Gamma^{0} \cap \mathcal{U}$ on which $U \rightarrow-\infty$. In the next chapter, we guarantee that $\Gamma \neq \varnothing$.

\section{COMPOSITIONS With QUASICONFORMAL TRANSFORMATIONS}

In this section $g$ will be transformed by means of compositions with quasiconformal mappings to produce a quasiregular function $F$ with asymptotic values precisely $A^{*}$. Recall that $A=A^{*} \backslash\{\infty\}$ (1) is analytic, and until $\S 8.2 A \subset B(0,2)$.

An analytic set $A$ is obtained from Lusin's operations:

$$
A=\bigcup_{\mathbb{N}^{\mathbb{N}}} \bigcap_{p \geq 1} \mathcal{S}_{n_{1}, \ldots, n_{p}}
$$

where the sets $\mathcal{S}_{n_{1}, \ldots, n_{p}}$ are closed (see [3] or [16], p. 207) and $\mathbb{N}^{\mathbb{N}}$ is the collection of infinite sequences of (positive) natural numbers. Sierpinski calls $A$ the nucleus of the system $\mathcal{S}_{n_{1}, \ldots, n_{p}}$.

We need a very precise description of the sets $\mathcal{S}_{n_{1}, \ldots, n_{p}}$, and the situation is complicated since different authors often use different definitions. Our formulation uses the ideas of [16, Thm. 112] but our condition 2), which is indispensable here, is slightly different than in [16] and does not appear in [3]. For convenience, we sketch a proof, and refer the reader to [16], $\S 86$ for full details.

Let $\mathcal{N}_{0}$ be the collection of all finite sequences $\left(n_{1}, \ldots, n_{p}\right)$.

Theorem A. Let $A \subset \mathbb{C}$ be a nonempty analytic set in $\mathbb{C}$, and let a decreasing positive sequence $\left\{\delta_{p}\right\}, \delta_{p} \downarrow 0$ be given. Then we may write $A$ as in (52) where

1) each $\mathcal{S}_{n_{1}, \ldots, n_{p}}$ is a closed set,

2) $\operatorname{diam}\left(\mathcal{S}_{n_{1}, \ldots, n_{p}}\right)<\delta_{p}$,

3) $\mathcal{S}_{n_{1}, \ldots, n_{p}, n_{p+1}} \subset \mathcal{S}_{n_{1}, \ldots, n_{p}}$

4) $\mathcal{S}_{n_{1}, \ldots, n_{p}} \neq \varnothing$ for all $\left(n_{1} \ldots, n_{p}\right)$ in $\mathcal{N}_{0}$.

Proof. The original definition in $\S 82$ of [16] uses only 1) and 3), and avoids 4). However, we are considering only nonempty analytic sets $A$. Thus for the moment assume that $A$ is as in (52), where only 1) and 3) hold; we call these sets $\mathcal{S}_{n_{1}, \ldots, n_{p}}^{\prime}$, and convert them to ones which satisfy 2 ) and 4 ) as well (in [16], $\delta_{p}=1 / p$ ). 
To secure 2), let the $\left\{\delta_{p}\right\}$ be given, and introduce for each $p$ a countable covering of $\mathbb{C}$ by closed balls $\left\{M_{n}^{(p)}\right\}$ of diameter $\delta_{p} / 2 \leq \operatorname{diam} M_{n}^{(p)}<\delta_{p}$. Then for each $n \in \mathbb{Z}$, take $\mathcal{S}_{n}^{o}=M_{n}^{(2)}$, and $\mathcal{S}_{n_{1}, n_{2}}^{o}=\mathcal{S}_{n_{1}}^{o}=M_{n_{1}}^{(2)}$. This is augmented for $p>1$ by

$$
\mathcal{S}_{n_{1}, n_{2}, \ldots, n_{2 p}}^{o}=\mathcal{S}_{n_{1}, n_{2}, \ldots, n_{2 p-1}}^{o}=\mathcal{S}_{n_{2}, n_{4}, \ldots, n_{2 p-2}}^{\prime} \cap M_{n_{2 p-1}}^{(2 p)},
$$

where $\left(n_{1}, n_{2}, \ldots, n_{2 p}\right)$ range over $\mathcal{N}_{0}$. It is clear that the sets $\mathcal{S}^{o}$ are closed, and easy to check that the nucleus of $\mathcal{S}^{o}$ coincides with that of $\mathcal{S}^{\prime}$. Thus 2) is satisfied.

Property 4) may be arranged as in [16], §86. Since $A \neq \varnothing$, choose some fixed $\omega_{0} \in A$. Then for any combination of $k$ indices, $m(k) \in \mathcal{N}_{0}$, and any sequence $\left(n_{1}, n_{2}, \ldots\right)$ of natural numbers, let $\mathcal{S}_{m(k), n_{1}, n_{2}, \ldots}^{o}=\cap_{p \geq 1} \mathcal{S}_{m(k), n_{1}, n_{2}, \ldots, n_{p}}^{o}$, and set

$$
\mathcal{S}_{m(k)}^{*}=\cup_{\mathbb{N}^{\mathbb{N}}} \mathcal{S}_{m(k), n_{1}, n_{2}, \ldots}^{o} .
$$

Sets of this nature must be included in (52). Whenever $\mathcal{S}_{m(k)}^{*} \neq \varnothing$, define $\mathcal{S}_{m(k)}=$ $\overline{\mathcal{S}_{m(k)}^{*}}$. However when $\mathcal{S}_{m(1)}^{*}=\varnothing$, set $\mathcal{S}_{m(1)}=\omega_{0} \in A($ since $A \neq \varnothing)$, and if $k_{0}+1$ is the least integer with $\mathcal{S}_{m(k)}^{*}=\varnothing$, set $\mathcal{S}_{m(j)}=\omega_{m\left(k_{0}\right)} \in \mathcal{S}_{m\left(k_{0}\right)}^{*}, j>k_{0}$.

The set of asymptotic values $\{0, \infty\}$ will be transformed into $A^{*}$ by successive compositions with quasiconformal transformations. Recall that a homeomorphism $\varphi$ is said to be $K$-quasiconformal $(K \geq 1)$ in $\mathbb{C}$ if it is in the Sobolev space $\varphi \in$ $W_{\text {loc }}^{1,2}(\mathbb{C})$ and its (formal) derivatives satisfy $\left|\varphi^{\prime}(z)\right|^{2} \leq K J_{\varphi}(z)$ a.e. $z \in \mathbb{C}$, where $J_{\varphi}$ is the Jacobian determinant (see [1] for more properties).

The sequence $\left\{\delta_{p}\right\}$ in Theorem A arises from repeated use of an elementary lemma on quasiconformal mappings (known to Teichmüller and proved in [1], see also [4]). The various choices of $\{R, \delta\}$ depend on the sets (52).

Lemma A. Let $2>K>1$ and $R>\delta$ be given. Consider the $(K, \delta, R)$ problem of finding a quasiconformal self-mapping of $\mathbb{C}, \varphi$, such that

1) $\varphi(w)=w$ if $|w| \geq R$,

2) for any given $\alpha$ such that $|\alpha| \leq \delta$, we have $\varphi(w)=w+\alpha$ if $|w| \leq \delta$,

3) $\varphi$ is K-quasiconformal.

Then, given either $R$ or $\delta$, there are choices of $\delta=\delta(R)$ or $R=R(\delta)$ which solve the problem.

We use this lemma in an iterative way. For a given $K>1$, take a sequence $K_{0}>K_{1}>\cdots$ with

$$
\prod_{j} K_{j}<K
$$

(thus $K_{j} \downarrow 1$ (very) rapidly). Apply Lemma A with $\delta=\delta_{0}=2$ and $K=K_{0}$, thus obtaining $R_{0}$, and for $j \geq 1$ take $K=K_{j}, R_{j}=\delta_{j-1}$ to obtain $\delta_{j} \downarrow 0$. The point $\alpha_{0}$ and in general, $\alpha_{j}(j \geq 0)$ will be specified later in section $\S 6$. It is convenient to assume, if necessary by decreasing $\delta_{j-1}$ at each appearance, that

$$
\log R_{j}+10 C_{0}<\log R_{j-1} \quad(j \geq 1),
$$

where $C_{0}$ is from Theorem 2. 
As in [5], this lemma will produce a large collection of quasiconformal mappings, all applied to $g(z)$ from Theorems 2 and 3. At each point $z$, the final quasiconformal mapping $\Psi$ will have at $w=g(z)$ the form

$$
\Psi(w)=\cdots \varphi_{j} \circ \cdots \varphi_{1} \circ \varphi_{0}(w)
$$

where $\varphi_{j}$ is $K_{j}$-quasiconformal mapping of $\mathbb{C}$, so that

$$
F(z)=\Psi \circ g(z)
$$

is a continuous $K$-quasiregular mapping (which unlike a $K$-quasiconformal mapping it need not to be a homeormorphism, see [15]). The functions $\left\{\varphi_{j}\right\}$ are related to the desired behavior of $F$ on a given branch $\gamma \subset \Gamma \subset \Gamma^{0} \subset \Gamma^{\sharp} \cap \mathcal{U}$ (recall Figure 2), with $\Gamma$ introduced at the end of $\S 4.4$. (We are simplifying notation, since in principle there should be different subscripts corresponding to each group of mappings in (54) associated to different paths $\gamma$. However the data $\left\{\delta_{j}, R_{j}, K_{j}\right\}$ is the same for each choice of $\varphi_{j}$.)

Thus let $\gamma \subset \Gamma \subset \Gamma^{0} \subset \mathcal{U}$ be a path on which $z \rightarrow \infty$ and $g(z) \rightarrow 0$. We arrange the $\left\{\varphi_{j}\right\}$ and $a_{n} \rightarrow a, a_{n} \in A$, so that the orbit of $w=0$ under $F$ as $z$ passes through $\gamma$ will be

$$
0 \rightarrow \varphi_{0}(0) \rightarrow \varphi_{1}\left(\varphi_{0}(0)\right) \rightarrow \cdots
$$

leading to $F(z) \rightarrow a=\lim a_{n}$ as $z \rightarrow \infty, z \in \gamma$.

There is a natural way to correspond each path $\gamma \subset \Gamma$ to a point of the set $A$ of (1), where $\Gamma \subset\left(\Gamma^{0} \cap \mathcal{U}\right)$ has been introduced at the end of $\S 4$. 4 . Each node of $\Gamma^{0} \cap \mathcal{U}$ will be associated to a specific point $a \in A$ using Theorem A. Since $\Gamma^{0} \cap \mathcal{U}$ is combinatorially a dyadic tree, its nodes correspond in a natural way to finite sequences of 0 's and 1's with first entry 0 . Let $\mathcal{B}$ be the countable collection of all such sequences. For each $m, \mathcal{B}$ has $2^{m}$ elements having $m$ entries after the first 0 . In turn, each such $b$ has two successors $b^{\prime}$ and $b^{\prime \prime}$ with $m+1$ entries after the first 0: their first $m$ entries coincide with those of $b$, and the final entry is 0 or 1 . This leads to the standard binary graph $\mathcal{G}$ associated with $\mathcal{B}$. Following [16], we associate a finite sequence $\left(n_{1}, n_{2} \ldots, n_{p}\right) \in \mathcal{N}_{0}$ to each $b \in \mathcal{B} \backslash\{0\}$, so that each node in a dyadic tree corresponds either to 0 or to a (unique) finite sequence of natural numbers. Let $b \in \mathcal{B}$. Then $b=0$ and $b=0.0 \ldots 0$ correspond to the number 0 . Otherwise, $b=0 . \xi_{1} \cdots \xi_{j}$, where $\xi_{i} \in\{0,1\}, 1 \leq i \leq j$, and at least one $\xi_{i} \neq 0$, corresponds to $\left(n_{1}, \ldots, n_{k}\right) \in \mathcal{N}_{0}$, where

$$
\sum_{i=1}^{j} \frac{\xi_{i}}{2^{i}}=\sum_{\ell=1}^{k} \frac{1}{2^{n_{1}+\cdots+n_{\ell}}} .
$$

This correspondence is coherent in the sense that if $b^{\prime}$ is has the same binary expansion as $b$ through the first $\ell$ appearances of 1 , then the first $\ell$ digits of $\left\{n_{1}, \ldots, n_{k}\right\}$ and $\left\{n_{1}, \ldots, n_{k^{\prime}}\right\}$ coincide.

In this way, every node of a dyadic tree is associated with a finite sequence of natural numbers or zero, and conversely, any finite sequence of natural numbers is associated to countably many nodes in a dyadic tree.

Once we have this correspondence, it is natural to exhaust $\mathcal{N}_{0}$ in the order induced by the tree structure of $\mathcal{B}$ :

$$
0 ; 0.0,0.1 ; 0.00,0.01,0.10,0.11 ; 0.000,0.001, \ldots,
$$


which produces the $\xi_{i}$ in (57). Thus if $k \geq 1$, the $k$-th bifurcation node $z_{k} \in \Gamma^{\sharp} \cap \mathcal{U}$ (see $\S 2$ and Figure 2) corresponds to the $k$-th new element in this display of $\mathcal{B}$; this is a number from (57) with 1 as final entry. In turn, (57) associates this node to a set $S_{n_{1}, \ldots, n_{p}}$ in the system (52). The specific mappings $\left\{\varphi_{j}\right\}$ chosen below reflect the data (52) as well as $\left\{R_{j}\right\}$ from Lemma A and $C_{0}$ from Theorems 2 and 3.

The connection between (58) and the evolution of $\Gamma^{\sharp}$ through bifurcations can be made concrete, in that at a bifurcation node $z_{k} \in S\left(r_{k}\right) \cap \mathcal{U}$ the new branch of $\Gamma^{0}$ (which corresponds to an element of $\mathcal{B}$ with last digit one), originating at $z_{k}$ is the arc of $\Gamma^{0}$ having larger argument. The curves $\gamma \in \Gamma \subset\left(\Gamma^{0} \cap \mathcal{U}\right)$ on which $U$ tends to $-\infty$ will be paths which have infinitely many segments corresponding to elements in $\mathcal{B}$ of (58) having terminal digit one (see (61), which then applies for infinitely many $p$ ). On these curves on which $g \rightarrow 0$ (see proof of Lemma 9) are where $F$ (and later $f$ ) attains asymptotic values $a \in A$.

We now define $U$ at the $\left\{z_{k}\right\}$ and use the procedure (16) to extend $U$ to the arcs of $\Gamma^{0}$ and then (3) and (4) to define $U$ on all of $\mathbb{C}$.

Start with $z_{0}=i r_{0}($ recall (10)), the first node corresponding to $0 \in \mathcal{B}$ and define

$$
U\left(z_{0}\right)=\log R_{0}+4 C_{0} .
$$

Note that other nodes $z_{p}$ that correspond to $0.0 \ldots 0 \in \mathcal{B}$ will appear on each $S\left(r_{k}\right)$, $k \geq 1$ as in (58).

In fact once an arc of $\Gamma^{\sharp} \cap \mathcal{U}$ is assigned to $\Gamma^{*}$, the locus of local maxima, it never is subject to bifurcation as $|z| \rightarrow \infty$. To complete the definition of $U$ on these 'free arcs' $\gamma^{\sharp} \subset \Gamma^{*} \cap \mathcal{U}$, we observe that its initial point lies at some bifurcation node $z_{k}(k>0)$, where $U$ will be defined in a moment (see (61)). As we follow along $\gamma^{\sharp}$ and encounter $z_{k \ell}=\gamma^{\sharp} \cap S\left(r_{k+\ell}\right)(\ell \geq 1)$, we require that

$$
U\left(z_{k \ell}\right) \geq U\left(z_{k}\right)+\ell,
$$

and so we obtain infinitely many curves $\gamma^{\sharp} \subset \mathcal{U}$ on which $U \rightarrow \infty$.

In general, if the node $z_{k}(k \geq 1)$ corresponds to $b_{k} \in \mathcal{B}$ and $n(p)=\left(n_{1}, \ldots, n_{p}\right)$ is the sequence of natural numbers associated to $b_{k}$ by (57), we define

$$
U\left(z_{k}\right)=\log R_{p}+2 C_{0},
$$

which we copy at any successor $z^{\prime}$, which corresponds to $b^{\prime}$ (itself a successor of $\left.b_{k}\right)$ associated to the same sequence $n(p)=\left(n_{1}, \ldots, n_{p}\right) \in \mathcal{N}_{0}$. In this way we also obtain countably many curves in $\Gamma^{0}$ on which $|U|$ does not have $\infty$ as an asymptotic value; on these $g$ will have no asymptotic value, as suggested at the end of $\S 2.1$. On the other hand, the curves for which (61) for an increasing sequence of infinitely many $p$ 's are the ones that conform $\Gamma$, where $U \rightarrow-\infty$.

\section{THE FAMILIES OF QUASICONFORMAL MAPPINGS.}

It follows from (52) that to any $a \in A$ corresponds a sequence $\left(n_{1}, n_{2}, n_{3}, \ldots\right)$ so that $a=\cap_{p=1}^{\infty} \mathcal{S}_{n_{1}, \ldots, n_{p}}$. We have already selected the $\left\{R_{p}, \delta_{p}\right\}$ in Lemma A.

Next, we identify the specific quasiconformal compositions $\left\{\varphi_{j}\right\}$ and the domains in which they act, all of which are in $\mathcal{U}$.

For each $n$ consider $\Omega_{n}$, the unbounded components of $\left\{|g(z)|<R_{n}\right\}$ that intersect $\Gamma^{\sharp} \cap \mathcal{U}$. Then each path $\gamma \subset \Gamma$ (on which $g \rightarrow 0$ ) passes through components $\mathcal{D}_{n}$ of $\Omega_{n}$ for each $n \geq 0$. Theorems 2 and 3 with (59) show that $|g(z)|>R_{0}$ on the arcs of $\Gamma^{*}$ contained in $\left\{|z|>r_{0}\right\}$ which meet at the node $z_{0}$ : thus $\Omega_{0} \subset \mathcal{U}$, and $\Omega_{0}$ is separated from $\partial \mathcal{U}$ by arcs of $\Gamma^{*}$. 
Similar considerations show that two components $\mathcal{D}_{n}^{i}$ and $\mathcal{D}_{n}^{j}$ are separated by arcs of $\Gamma^{\sharp}$.

In general each component $\mathcal{D}_{n}^{m}$ of $\Omega_{n}$ will contain countably many components $\mathcal{D}_{n+1}^{j}$ of $\Omega_{n+1}$, each of which will contain countably many disjoint components $\mathcal{D}_{n+2}^{\ell}$ of $\Omega_{n+2}, \ldots$, imitating the process $(52)$.

It is in these domains $\mathcal{D}_{n}$ that we introduce the mappings $\varphi_{n}$. Consider a nested chain of sets

$$
\mathcal{D}_{0}^{n_{1}} \supset \mathcal{D}_{1}^{n_{2}} \supset \mathcal{D}_{2}^{n_{3}} \supset \cdots ;
$$

these sets will then contain the asymptotic path $\gamma$ at which the asymptotic value $a=\cap_{k=1}^{\infty} \mathcal{S}_{n_{1}, \ldots, n_{k}}$ will be attained.

The quasiregular mapping $F$ is defined on each chain $\mathcal{D}_{p}^{n_{p+1}}$ inductively in the domains $\overline{\Omega_{p}} \backslash \Omega_{p+1}$. First, take $F(z)=g(z)$ if $z \in \mathbb{C} \backslash \Omega_{0}$, observing from (59) and the role of $C_{0}$ in Theorem 2 that since $\left|g\left(z_{0}\right)\right|>\log R_{0}+2 C_{0}, z_{0}$ is not in $\Omega_{0}$. Fix $n \in \mathbb{N}$ and consider a domain $\mathcal{D}_{0}^{n} \subset \Omega_{0}$. We set

$$
F(z)=\varphi_{0} \circ g(z), \quad z \in \overline{\mathcal{D}_{0}^{n}} \backslash \Omega_{1},
$$

where $\varphi_{0}$ is the $K_{0}$-quasiconformal map given by Lemma A (which produced the original $R_{0}$ ), so that $\varphi_{0}(w)=w$ if $|w| \geq R_{0}$ and $\varphi_{0}(w)=w+a_{1}$ if $|w| \leq \delta_{0}$, where $a_{1} \in A \cap \mathcal{S}_{n}$ as in (52), with (cf. Theorem A) diam $\mathcal{S}_{n}<\delta_{1}$. Thus, if $z \in \partial \Omega_{1} \subset \mathcal{D}_{0}^{n}$ then $|g(z)|=R_{1}=\delta_{0}$ and therefore $F(z)=g(z)+a_{1}$ (so by means of $\varphi_{0}, g(z)$ has been translated to $g(z)+a_{1}$, the first step of the chain (56)). More important, since $a_{1} \in A \cap \mathcal{S}_{n}$, properties 2) and 3) of Theorem A ensure that when $\varphi_{1}$ is introduced as in (54), all possible choices $a_{2} \in \mathcal{S}_{n, m} \subset \mathcal{S}_{n}$ satisfy

$$
\left|a_{2}-a_{1}\right|<\delta_{1}
$$

Notice that $F$ is well-defined and continuous in $\mathbb{C} \backslash \Omega_{1}$. Indeed, for $m \neq n$, let $\mathcal{D}_{0}^{m}$ be another component of $\Omega_{0}$. Then

$$
F(z)=\varphi_{0}^{\prime} \circ g(z), \quad z \in \overline{\mathcal{D}_{0}^{m}} \backslash \Omega_{1}
$$

where $\varphi_{0}^{\prime}$ is the $K_{0}$-quasiconformal map given by Lemma $\mathrm{A}$ with $\varphi_{0}^{\prime}(w)=w$ if $|w| \geq R_{0}$ and $\varphi_{0}(w)=w+a_{1}^{\prime}$ if $|w| \leq \delta_{0}$, where $a_{1}^{\prime} \in A \cap \mathcal{S}_{m}$. We have already checked that $\mathcal{D}_{0}^{m} \cap \mathcal{D}_{0}^{n}=\varnothing$. Thus $F$ is well-defined in $\mathbb{C} \backslash \Omega_{1}$ and continuous on $\partial \mathcal{D}_{0}^{n}$ since if $z \in \partial \mathcal{D}_{0}^{n}$ then $|g(z)|=R_{0}$ and therefore $F(z)=g(z)$.

Suppose that $F$ is defined in $\mathbb{C} \backslash \Omega_{p}$, now we show how to extend it to $\mathbb{C} \backslash \Omega_{p+1}$. Let $\mathcal{D}_{p-1}^{n_{p}} \subset \Omega_{p-1}$ be given and consider a domain $\mathcal{D}_{p}^{n} \subset \mathcal{D}_{p-1}^{n_{p}}$. Assume that

$$
F(z)=\Phi_{p-1} \circ g(z), \quad z \in \overline{\mathcal{D}_{p-1}^{n_{p}}} \backslash \Omega_{p},
$$

where $\Phi_{p-1}=\varphi_{p-1} \circ \cdots \circ \varphi_{0}$ is $K_{p-1}$-quasiconformal mapping chosen from Lemma A with data $R_{p-1}, \delta_{p-1}$, such that in particular

$$
F(z)=g(z)+a_{p}, \quad z \in \partial \Omega_{p} \subset \mathcal{D}_{p-1}^{n_{p}},
$$

and $a_{p}$ is in $\mathcal{S}_{n_{1}, \ldots, n_{p}}$, a set of diameter less than $\delta_{p}$. In $\mathcal{D}_{p}^{n}$ define

$$
F(z)=\Phi_{p} \circ g(z), \quad z \in \overline{\mathcal{D}_{p}^{n}} \backslash \Omega_{p+1},
$$

where $\Phi_{p}$ is a quasiconformal mapping defined by $\Phi_{p}=\varphi_{p} \circ \Phi_{p-1}$ and $\varphi_{p}$ is a function given by Lemma A with $K=K_{p}$,

$$
\varphi_{p}(w)=w, \quad \text { when } \quad\left|w-a_{p}\right| \geq R_{p}
$$

and

$$
\varphi_{p}(w)=w+a_{p+1}-a_{p}, \quad \text { when } \quad\left|w-a_{p}\right| \leq \delta_{p}
$$


$a_{p+1} \in \mathcal{S}_{n_{1}, \ldots, n_{p}, n}$ and $\varphi_{p}$ is well defined since $a_{p+1}$ and $a_{p}$ lie in $\mathcal{S}_{n_{1}, \ldots, n_{p}}$, a set of diameter less than $\delta_{p}$.

The function $F$ is well-defined in $\mathbb{C} \backslash \Omega_{p+1}$ since the domains $\mathcal{D}_{p}^{k}$ and $\mathcal{D}_{p}^{n} k \neq n$ are disjoint, again using an appeal to (60).

Moreover, $F$ is continuous on $\partial \Omega_{p}$. Let $z \in \partial \mathcal{D}_{p}^{n} \subset \mathcal{D}_{p-1}^{n_{p}}$, then $|g(z)|=R_{p}=$ $\delta_{p-1}$ and by (62) and the definition of the function $\varphi_{p}$,

$$
F(z)=\Phi_{p-1}(g(z))=g(z)+a_{p}=\Phi_{p}(g(z)) .
$$

Finally, to verify (62) in these domains, consider a domain $\mathcal{D}_{p+1}^{\ell}$ contained in $\mathcal{D}_{p}^{n}$ and let $z \in \partial \mathcal{D}_{p+1}^{\ell} \subset \mathcal{D}_{p}^{n}$. Then $|g(z)|=R_{p+1}=\delta_{p}$ and

$$
F(z)=\varphi_{p}\left(g(z)+a_{p}\right)=g(z)+a_{p+1} .
$$

Thus $a=\cap_{p \geq 1} \mathcal{S}_{n_{1}, \ldots, n_{p}}$ is an asymptotic value of $F$, obtained on the path $\gamma$ passing through the domains $\mathcal{D}_{0}^{n_{1}} \supset \mathcal{D}_{1}^{n_{2}} \supset \mathcal{D}_{2}^{n_{3}} \supset \cdots$.

\section{Solution of Theorem 1}

\subsection{Nevanlinna Characteristic of $g$.}

Theorem 5. The meromorphic function g has order zero. Indeed,

$$
T(r, g)=o\left(\psi(r) \log ^{2} r\right) \quad(r \rightarrow \infty) .
$$

The Nevanlinna theory for subharmonic functions is discussed in [10] and adapts readily to $\delta$-subharmonic functions. We first estimate the counting-function for the 'poles' in $B(r), n(r, u)$. Formula (17) shows that the number of poles on any branch of $\Gamma^{\sharp} \cap B(r)$ is at most $L(r) \log r$, and (9) asserts that the number of branches in $B(r)$ is $O\left(L^{1 / 3}(r)\right)$. This means that

$$
n(r, g)=O\left(L^{4 / 3}(r) \log r\right) .
$$

Since $L$ increases and $E$ has density zero, we may integrate:

$$
N(r, g)=O\left(L^{4 / 3}(r) \log ^{2} r\right) .
$$

To estimate $T(r, g)=m(r, g)+N(r, g)$ we consider the proximity function,

$$
m(r, g)=\frac{1}{2 \pi} \int_{0}^{2 \pi} \log ^{+}\left|g\left(r e^{i \theta}\right)\right| d \theta .
$$

By Theorem $2, \log ^{+}|g(z)|=O(L(|z|))$ when $|z| \rightarrow \infty$ and $z \notin E$, so it is enough to check the contribution to $m(r, g)$ from integration over the exceptional set $E$.

However the estimate is then routine given the representation (38) of each $h_{\mathbf{J}}$, since we may perform an explicit integration over each of the of disks of $E \cap S(r)$ for each $r$ with $S(r) \cap E \neq \varnothing: m(r, g)=O(L(r)$ ). Thus (on recalling (8))

$$
T(r)=(1+o(1)) N(r, g)=O\left(L^{4 / 3}(r) \log ^{2} r\right)=o\left(\psi(r) \log ^{2} r\right) \quad(r \rightarrow \infty) .
$$

(Alternatively, since $T(r)=o\left(\psi(r) \log ^{2} r\right.$ ) when $S(r) \cap E=\varnothing$, we obtain it for the remaining $r$ since $T$ increases.) 
7.2. Asymptotic values of $F$. We return to the function $F$ which was obtained in $\S 5$. Recall that we still assume that $A=A^{*} \backslash\{\infty\}$ and $A \subset B(0,2)$.

Lemma 10. The asymptotic values of $F$ are $w=0, w=\infty$ and values a which are limits of $g(z)$ on curves $\gamma \subset \Gamma^{0} \cap \mathcal{U}$. In particular, $A s(F)=A \cup\{\infty\}=A^{*}$.

Proof. This depends on the form of the compositions (54) and (55) along with Theorem 4. Note that $\{0, \infty\} \subset A s(F)$ since there are many curves in $\Gamma^{\sharp}$ in the lower half-plane on which $F \rightarrow 0, \infty$, with no other asymptotic values.

We first show that only asymptotic values associated by the procedure of $\S 5$ are asymptotic values of $F$. Let $F(z) \rightarrow a$ on $\eta$. Once we show that $g(z)$ itself has a limit $a^{\prime}$ on $\eta$, Theorem 4 shows that $a^{\prime}=0$ or $a^{\prime}=\infty$. Since all compositions $\Psi$ are the identity outside $B\left(R_{0}\right)$, we certainly have $a^{\prime}=\infty$ when $a=\infty$.

Thus suppose $|a|<R_{0}$. Given $\delta>0$, choose $r^{\prime}>0$ so that $|F(z)-a|<\delta$ for $z \in \eta\left(r^{\prime}\right)$ with $\eta\left(r^{\prime}\right)$ the unbounded component of $\eta \cap\left\{|z|>r^{\prime}\right\}$.

The family of $K$-quasiconformal homeomorphisms of the sphere which fix $B\left(R_{0}\right)$ are uniformly Hölder continuous. Hence if $\Psi$ is any fixed function of the class (54), any $\Psi^{-1}$ image of $B(a, \delta)$ is contained in $B\left(\Psi^{-1}(a), C^{\prime} \delta^{\alpha}\right)$, with $\alpha=\alpha(K)$.

It follows that if $\Psi^{\prime}$ is a choice of $\Psi$ at $g\left(z^{\prime}\right)$, with $z^{\prime} \in \eta \cap S\left(r^{\prime}\right)$, then $g(z) \in$ $B\left(\Psi^{\prime-1}\left(F\left(z^{\prime}\right)\right), C^{\prime} \delta^{\alpha}\right)$. Since $\delta \rightarrow 0$ as $r^{\prime} \rightarrow \infty$ and the family of functions $\{\Psi\}$ is normal, $g$ itself must have a limit on $\eta$. As we showed in $\S 4.2$, this means that $\eta$ is contained in a tract on which $g \rightarrow 0$, so this tract also contains a curve $\gamma \subset \Gamma \subset \Gamma^{0}$. If $\gamma \subset \Gamma^{0} \cap \mathcal{U}$ then the choice of compositions in (54) was made so that $F(z) \rightarrow a \in A$ in $\gamma$, and so in $\eta$. If, on the other hand, $\gamma \subset \Gamma^{0}$ in the lower half plane, then $F(z)=g(z)$ on $\gamma$ and so $F(z) \rightarrow 0 \in A$ in $\gamma$, and therefore on $\eta$.

7.3. Construction of $f$; completion of proof. Let $F$ be from (55). The meromorphic function $f$ of Theorem 1 is obtained using standard techniques. Let $\sigma(z)=\left(F_{\bar{z}} / F_{z}\right)(z)$ and $f:=F \circ \tau^{-1}$ where $\tau$ is the homeomorphic solution to

$$
\tau_{\bar{z}}(z)=\sigma(z) \tau_{z}(z) \quad \text { (Beltrami equation), }
$$

normalized to fix 0,1 and $\infty$. Then $f$ is meromorphic in the plane.

Obviously $A s(f)=A^{*}$, so we need only check (2).

We may avoid delicate distortion theorems on solutions to the Beltrami equation, since $g$ is of slow growth (cf. (2)). A standard distortion theorem [1] (Hölder continuity) gives that if $w=\tau(z)$ satisfies this equation with $\|\sigma\|_{\infty}<\kappa<1$, then there are $A=A(\kappa), M=M(\kappa)$ with

$$
|\tau(z)|<A|z|^{M} \quad(z \in \mathbb{C}) .
$$

Lemma 11. The characteristic of the meromorphic function $f$ satisfies (2).

Proof. Since all quasiconformal compositions used in the previous section fix a neighborhood of $w=\infty$, we have $n(r, \infty, F) \equiv n(r, \infty, g)(r>0)$. We may suppose that $K$ in (53) has been taken so that (63) holds with $M \leq 20$, and so $n(r, f) \leq$ $n\left(C r^{20}, g\right)=O\left(C L^{4 / 3}\left(r^{20}\right)\right) \log ^{2} r$. Using (8), we find

$$
N(r, f):=\int^{r} t^{-1} n(t, f) d t \leq N\left(21 C L^{4 / 3}\left(r^{21}\right)\right)=o\left(\psi(r) \log ^{2} r\right) .
$$

Similarly, $m(r, f)=O\left(L\left(A r^{M} g\right)\right)$, and since $T(r, f)=m(r, f)+N(r, f)$, a final appeal to (8) gives (2). 


\section{CONCLUding REMARKS}

In this section, we settle some loose ends.

8.1. Functions of given order $\lambda$. To construct functions of order $\lambda \neq 0$ requires a simple trick (we thank A. Eremenko for this suggestion). Let $g$ be the meromorphic function (of order zero) just constructed, with $A s(g)=\{0, \infty\}$. Let $W$ be an unbounded open set with $d\left(W, \Gamma^{\sharp}\right)>1$. Choose a sequence $\left\{w_{n}\right\}$ tending to $\infty$ in $W$ whose exponent of convergence is $\lambda$ (for example, let the number of $w_{n}$ in $B(r$ ) be asymptotic to $\left.r^{\lambda}\right)$.

Next, for each $w_{n}$ choose $b_{n}$ with $w_{n}-b_{n}$ tending so rapidly to zero that

$$
\Pi(z)=\frac{1-z / w_{n}}{1-z / b_{n}}
$$

is so close to one outside $W$ that if $g_{1}(z)=g(z) \Pi(z)$, then $g-g_{1}=o(1)$ and $\arg g(z)-\arg g_{1}(z)=o(1)$ as $z \rightarrow \infty$ in a neighborhood of $\Gamma^{\sharp}$. Then $g_{1}$ has order $\lambda$, and we may perform the compositions of $\S 5$ on $g_{1}$, yielding $f_{1}$ of order $\lambda$ with $A^{*}$ its asymptotic set.

8.2. General analytic sets $A^{*}$. To remove the assumption that the set $A$ of (1) be contained in $B(0,2)$, we construct a 'forest' of trees in $\mathcal{U}$. Thus, instead of $\Gamma^{0} \subset \Gamma^{\sharp}$ being a single tree beginning on the positive imaginary axis (cf $\S 2.3$ ), there will be a countable collection of trees $\Gamma_{m, n} \subset \mathcal{U}, \Gamma_{m, n} \subset \Gamma^{\sharp}$ with asymptotic values being those of $A \cap B(m+n i, 2)$.

Since the each of the compositions in (54) operates in disjoint regions of the plane, the proofs of Lemma 10 and Lemma 11 apply as before.

\section{REFERENCES}

[1] L. Ahlfors, Lectures on Quasiconformal mappings: Second edition. With supplemental chapters by C. J. Earle, I. Kra, M. Shishikura and J. H. Hubbard, University Lecture Series, vol. 38, American Mathematical Society, Providence, 2006. MR2241787

[2] A. Baernstein, Proof of Edrei's spread conjecture, Proc. London Math. Soc. 26 (1973), no. 3, 418-434. MR0374429

[3] L. Carleson, Selected Problems on Exceptional Sets, Van Nostrand, Princeton, N.J.-Toronto, Ont.-London, 1967. MR0225986

[4] D. Drasin, The inverse problem of the Nevanlinna theory, Acta Math. 138 (1976), 83-151. MR0585644

[5] On a method of Holopainen and Rickman, Israel J. of Mathematics 101 (1997), 73-84. MR1484869

[6] - Approximation of subharmonic functions with applications, Complex Analysis, and Potential Theory (Montreal, Canada, 2000), Proceedings of the NATO ASI, vol. 37, Kluwer Acad. Publ., Dordrecht, 2001, pp. 163-189. MR1873588

[7] D. Drasin and J.M. Wu, The sharpness of a criterion of MacLane for the class $\mathcal{A}$, J. London Math. Soc. 67 (2003), no. 2, 433-447. MR1956145

[8] A. Eremenko, The set of asymptotic values of a finite order meromorphic function, Mat. Zametki 24 (1978), 779-783; English transl., Math. Notes 24 (1978), no. 5-6, 914-916. MR0522410

[9] M.A. Girnyk, On the approximation of a subharmonic function of infinite order by the logarithm of the modulus of an entire function, Mat. Zametki 50 (1991), no. 4, 57-60; English transl., Math. Notes 50 (1991), no. 3-4, 1025-1027. MR1162911 
[10] W.K. Hayman and P.B. Kennedy, Subharmonic Functions, Vol. 1, Academic Press, New York, 1976. MR0460672

[11] M. Heins, The set of asymptotic values of an entire function, Tolfte Skandinaviska Matematikerkongressen (Lund, Sweden, 1953), Proceedings of the Scandinavian Math. Congress, Lund, 1954, pp. 56-60. MR0067989

[12] Y. Lyubarskii and E. Malinnikova, On approximation of subharmonic functions, J. Anal. Math. 83 (2001), 121-149. MR1828489

[13] S. Mazurkiewicz, Sur les points singuliers d'une fonction analytique, Fund. Math. 17 (1931), $26-29$.

[14] J. Ortega-Cerdà, Multipliers and weighted $\bar{\partial}$-estimates, Rev. Mat. Iberoamericana 18 (2002), no. 2, 355-377. MR1949832

[15] S. Rickman, Quasiregular mappings, Ergebnisse der Mathematik und ihrer Grenzgebiete [Results in Mathematics and Related Areas, vol. 26, Springer-Verlag, Berlin, 1993. MR1238941

[16] W. Sierpinski, Introduction to General Topology, University of Toronto Press, Toronto, 1934.

[17] G. Valiron, Sur les valeurs asymptotiques de quelques fonctions méromorphes, Rend. Circ. Mat. Palermo 49 (1925), 415-421.

[18] R.S. Yulmukhametov, Approximation of subharmonic functions, Anal. Math. 11 (1985), no. 3, 257-282 (Russian. English summary). MR0822590

Debin, ETsi navales, Universidad Politécnica de Madrid, Av. Arco de la Victoria, 28040 MADRID, SPAIN

E-mail address: alicia.canton@upm.es

Mathematics Department Purdue University. West Lafayette, IN 47907, USA

E-mail address: drasin@math.purdue.edu

Department of Mathematics, Saint louis University, Madrid Campus; Av. del Valle 34, 28003 MADRID, SPAIN

E-mail address: agranado@slu.edu 